\title{
First detection of VHE gamma-ray emission from TXS 1515-273, study of its X-ray variability and spectral energy distribution
}

MAGIC Collaboration ${ }^{\star}$ : V. A. Acciari ${ }^{1}$, S. Ansoldi ${ }^{2}$, L. A. Antonelli ${ }^{3}$, A. Arbet Engels ${ }^{4}$, M. Artero ${ }^{5}$, K. Asano ${ }^{6}$, D. Baack ${ }^{7}$, A. Babić ${ }^{8}$, A. Baquero ${ }^{9}$, U. Barres de Almeida ${ }^{10}$, J. A. Barrio ${ }^{9}$, I. Batković ${ }^{11}$, J. Becerra González ${ }^{1}$, W. Bednarek ${ }^{12}$, L. Bellizzi ${ }^{13}$, E. Bernardini ${ }^{14}$, M. Bernardos ${ }^{11}$, A. Berti ${ }^{15}$, J. Besenrieder ${ }^{16}$, W. Bhattacharyya ${ }^{14}$, C. Bigongiari ${ }^{3}$, A. Biland ${ }^{4}$, O. Blanch ${ }^{5}$, Ž. Bošnjak ${ }^{8}$, G. Busetto ${ }^{11}$, R. Carosi ${ }^{17}$, G. Ceribella ${ }^{16}$, M. Cerruti ${ }^{18}$, Y. Chai ${ }^{16}$, A. Chilingarian ${ }^{19}$, S. Cikota ${ }^{8}$, S. M. Colak ${ }^{5}$, E. Colombo ${ }^{1}$, J. L. Contreras ${ }^{9}$, J. Cortina ${ }^{20}$, S. Covino ${ }^{3}$, G. D’Amico ${ }^{16}$, V. D'Elia ${ }^{3}$, P. Da Vela $^{17,38}$, F. Dazzi ${ }^{3}$, A. De Angelis ${ }^{11}$, B. De Lotto ${ }^{2}$, M. Delfino ${ }^{5,39}$, J. Delgado ${ }^{5,39}$, C. Delgado Mendez ${ }^{20}$, D. Depaoli ${ }^{15}$, F. Di Pierro ${ }^{15}$, L. Di Venere ${ }^{21}$, E. Do Souto Espiñeira $^{5}$, D. Dominis Prester ${ }^{22}$, A. Donini ${ }^{2}$, D. Dorner ${ }^{23}$, M. Doro ${ }^{11}$, D. Elsaesser ${ }^{7}$, V. Fallah Ramazani ${ }^{24,40, \star}$, A. Fattorini ${ }^{7}$, G. Ferrara ${ }^{3}$, M. V. Fonseca ${ }^{9}$, L. Font ${ }^{25}$, C. Fruck ${ }^{16}$, S. Fukami ${ }^{6}$, R. J. García López ${ }^{1}$, M. Garczarczyk ${ }^{14}$, S. Gasparyan ${ }^{26}$, M. Gaug ${ }^{25}$, N. Giglietto ${ }^{21}$, F. Giordano ${ }^{21}$, P. Gliwny ${ }^{12}$, N. Godinović ${ }^{27}$, J. G. Green ${ }^{3}$, D. Green ${ }^{16}$, D. Hadasch ${ }^{6}$, A. Hahn ${ }^{16}$, L. Heckmann ${ }^{16}$, J. Herrera ${ }^{1}$, J. Hoang ${ }^{9}$,

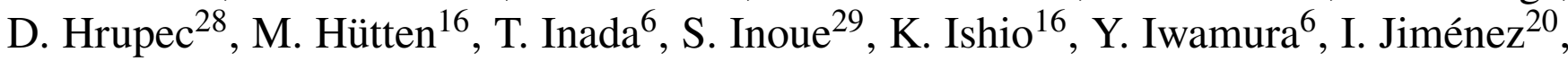
J. Jormanainen ${ }^{24}$, L. Jouvin ${ }^{5}$, Y. Kajiwara ${ }^{30}$, M. Karjalainen ${ }^{1}$, D. Kerszberg ${ }^{5}$, Y. Kobayashi ${ }^{6}$, H. Kubo ${ }^{30}$, J. Kushida ${ }^{31}$, A. Lamastra ${ }^{3}$, D. Lelas ${ }^{27}$, F. Leone ${ }^{3}$, E. Lindfors ${ }^{24, \star}$, S. Lombardi ${ }^{3}$, F. Longo ${ }^{2,41}$, R. López-Coto ${ }^{11}$, M. López-Moya ${ }^{9}$, A. López-Oramas ${ }^{1}$, S. Loporchio ${ }^{21, \star}$, B. Machado de Oliveira Fraga ${ }^{10}$, C. Maggio ${ }^{25}$, P. Majumdar ${ }^{32}$, M. Makariev ${ }^{33}$, M. Mallamaci ${ }^{11}$, G. Maneva ${ }^{33}$, M. Manganaro ${ }^{22}$, K. Mannheim ${ }^{23}$, L. Maraschi ${ }^{3}$, M. Mariotti ${ }^{11}$, M. Martínez ${ }^{5}$, D. Mazin ${ }^{6,42}$, S. Menchiari ${ }^{13}$, S. Mender ${ }^{7}$, S. Mićanović2 ${ }^{22}$, D. Miceli ${ }^{2}$, T. Miener ${ }^{9}$, M. Minev $^{33}$, J. M. Miranda ${ }^{13}$, R. Mirzoyan ${ }^{16}$, E. Molina ${ }^{18}$, A. Moralejo ${ }^{5}$, D. Morcuende ${ }^{9}$, V. Moreno ${ }^{25}$, E. Moretti ${ }^{5}$, V. Neustroev ${ }^{34}$, C. Nigro ${ }^{5}$, K. Nilsson ${ }^{24}$, K. Nishijima ${ }^{31}$, K. Noda ${ }^{6}$, S. Nozaki ${ }^{30}$, Y. Ohtani ${ }^{6}$, T. $\mathrm{Oka}^{30}$, J. Otero-Santos ${ }^{1}$, S. Paiano ${ }^{3}$, M. Palatiello ${ }^{2}$, D. Paneque ${ }^{16}$, R. Paoletti ${ }^{13}$, J. M. Paredes ${ }^{18}$, L. Pavletić ${ }^{22}$, P. Peñil ${ }^{9}$, C. Perennes ${ }^{11}$, M. Persic ${ }^{2,43}$, P. G. Prada Moroni ${ }^{17}$, E. Prandini ${ }^{11}$, C. Priyadarshi ${ }^{5}$, I. Puljak ${ }^{27}$, W. Rhode ${ }^{7}$, M. Ribó ${ }^{18}$, J. Rico ${ }^{5}$, C. Righi ${ }^{3}$, A. Rugliancich ${ }^{17}$, L. Saha ${ }^{9}$, N. Sahakyan ${ }^{26}$, T. Saito ${ }^{6}$, S. Sakurai ${ }^{6}$, K. Satalecka ${ }^{14}$, F. G. Saturni ${ }^{3}$, B. Schleicher ${ }^{23}$, K. Schmidt ${ }^{7}$, T. Schweizer ${ }^{16}$, J. Sitarek ${ }^{12}$, I. Šnidarićc ${ }^{35}$, D. Sobczynska ${ }^{12}$, A. Spolon ${ }^{11}$, A. Stamerra ${ }^{3}$, D. Strom ${ }^{16}$,

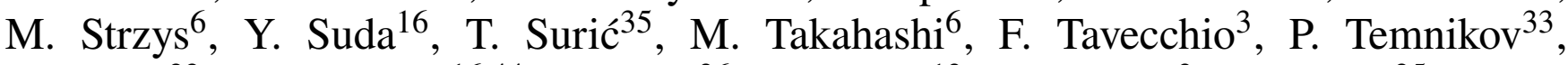
T. Terzić2 ${ }^{22}$, M. Teshima ${ }^{16,44}$, L. Tosti ${ }^{36}$, S. Truzzi ${ }^{13}$, A. Tutone ${ }^{3}$, S. Ubach ${ }^{25}$, J. van Scherpenberg $^{16}$, G. $\operatorname{Vanzo}^{1}$, M. Vazquez Acosta ${ }^{1}$, S. Ventura ${ }^{13}$, V. Verguilov ${ }^{33}$, C. F. Vigorito ${ }^{15}$, V. Vitale ${ }^{37}$, I. Vovk ${ }^{6}$, M. Will ${ }^{16}$, C. Wunderlich ${ }^{13}$, D. Zarić27, E. Bissaldi ${ }^{45,46}$, G. Bonnoli ${ }^{13,47}$, S. Cutini ${ }^{48}$, F. D’Ammando ${ }^{49}$, A. Nabizadeh ${ }^{50}$, A. Marchini ${ }^{51}$, and M. Orienti ${ }^{49}$ (affiliations are listed at the end of the paper) 


\begin{abstract}
We report here on the first multi-wavelength (MWL) campaign on the blazar TXS 1515-273, undertaken in 2019 and extending from radio to very-high-energy gamma rays (VHE). Up until now, this blazar had not been the subject of any detailed MWL observations. It has a rather hard photon index at $\mathrm{GeV}$ energies and was considered a candidate extreme high-synchrotronpeaked source. MAGIC observations resulted in the first-time detection of the source in VHE with a statistical significance of $7.6 \sigma$. The average integral VHE flux of the source is $6 \pm 1 \%$ of the Crab nebula flux above $400 \mathrm{GeV}$. X-ray coverage was provided by Swift-XRT, XMMNewton, and NuSTAR. The long continuous X-ray observations were separated by $\sim 9 \mathrm{~h}$, both showing clear hour scale flares. In the XMM-Newton data, both the rise and decay timescales are longer in the soft X-ray than in the hard X-ray band, indicating the presence of a particle cooling regime. The X-ray variability timescales were used to constrain the size of the emission region and the strength of the magnetic field. The data allowed us to determine the synchrotron peak frequency and classify the source as a flaring high, but not extreme, synchrotron peaked object. Considering the constraints and variability patterns from the X-ray data, we model the broad-band spectral energy distribution. We applied a simple one-zone model, which could not reproduce the radio emission and the shape of the optical emission, and a two-component leptonic model with two interacting components, enabling us to reproduce the emission from radio to VHE band.
\end{abstract}

Key words: galaxies: active - BL Lacertae objects: individual (TXS 1515-273) - Radiation mechanisms: non-thermal

\section{INTRODUCTION}

Almost all of the extragalactic sources detected above $100 \mathrm{GeV}$ are classified as Active Galactic Nuclei (AGNs): galaxies hosting a super-massive black hole in their centre whose gravitational potential energy is the ultimate source of the AGN luminosity. Up to $\sim 10 \%$ of AGNs develop two narrow jets of relativistic particles extending well outside the galaxy and emitting non-thermal radiation over the whole electromagnetic spectrum (Padovani et al. 2017). The spectra observed from jetted-AGNs depends strongly on the viewing angle of the jet with respect to the Earth, leading to their empirical classification. Jetted-AGNs with jets seen from large angles are classified as radio galaxies, while those seen at small viewing angles $\left(\theta<10^{\circ}-15^{\circ}\right)$ are known as blazars. Blazars' spectra are fully dominated by the jet emission, which can completely outshine the rest of the galaxy. They can be divided into flat spectrum radio quasars (FSRQs) and BL Lac objects (BL Lacs), depending on their optical spectra: FSRQs show strong, broad emission lines while BL Lacs display at most weak emission lines (Stickel et al. 1991; Stocke et al. 1991).

The broad-band spectral energy distribution (SED) of blazar emission is characterised by two distinct humps (Ghisellini et al. 2017). The first one peaks at infrared to $X$-ray frequencies and is commonly explained as due to synchrotron emission from ultrarelativistic electrons accelerated in the jet. The second hump, peaking above $\mathrm{MeV}$ energies, is most likely due to inverse Compton (IC) scattering, possibly of the same electrons on their own synchrotron emission (synchrotron self-Compton, SSC). The presence of a sub-dominant hadronic component is also possible, as discussed in Aharonian (2000) and Murase et al. (2012).

The energy of the synchrotron peak leads to a further subclassification of blazars. The peak frequency ranges from IR-optical to UV-soft-X bands in low, intermediate or high synchrotron-

* Send offprint requests to MAGIC Collaboration (e-mail: contact.magic@mpp.mpg.de). Corresponding authors are S. Loporchio, E. Lindfors, and V. Fallah Ramazani. peaked sources (LSP, ISP, HSP respectively, see Abdo et al. 2010). In particular, HSPs display a synchrotron emission peaking at frequencies $v_{X} \gtrsim 10^{15} \mathrm{~Hz}$. Furthermore, evidence for objects with synchrotron peak frequency exceeding the HSP soft-X ray band was found in Ghisellini (1999) and Costamante et al. (2001), with the most extreme high frequency-peaked blazars (EHBL) showing peaks above $10^{17} \mathrm{~Hz}$ (Costamante and Ghisellini 2002; Bonnoli et al. 2015) and a hard spectrum (photon index $\leq 2$ ) at the Fermi Large Area Telescope (LAT) energies, with the IC peak generally in the energy range above $100 \mathrm{GeV}$. The origin of such an extreme peak exceeding $\mathrm{TeV}$ energies and an explanation for the hard intrinsic spectrum at sub-TeV energies are still widely debated. This feature indicates the presence of a hard accelerated particle spectrum with most of the energy carried by the highest-energy particles.

As reported in Fossati et al. (1998), evidence of an empirical sequence connecting blazar classes with their bolometric luminosity was found, with LSP showing higher luminosity with respect to HSP blazars. The sequence was later revised by Ghisellini et al. (2017), finding good agreement with the original one. It is worth mentioning that the existence of the blazar sequence has been disputed ever since it was proposed, and it is still under debate (e.g. Keenan et al. 2020).

For any given blazar, only complete energy coverage, ranging from radio to $\mathrm{TeV}$ energies will allow for a robust study of the emission mechanisms. However, due to the variability of these objects, it is of crucial importance that these multi-wavelength (MWL) observations are performed simultaneously.

Although reported in the Second and the Third Catalog of AGNs detected by the Fermi-LAT (Ackermann et al. 2011, 2015), in the general Fermi-LAT catalogues (Nolan et al. 2012; Acero et al. 2015; Abdollahi et al. 2020) and in the high-energy catalogues (Ackermann et al. 2013, 2016; Ajello et al. 2017), the source TXS 1515-273 has been very little studied, and it has never been investigated intensively in the $\mathrm{X}$-rays before the observations reported in this work. An upper limit on its redshift $z<1.1$ was established in Kaur et al. (2018) with photometric methods. Recently, firm detection of spectral lines settled the redshift to $\mathrm{z}=0.1285$ (Goldoni et al. 2020; Becerra González et al. 2020). 
Table 1. Summary of the different observations performed.

\begin{tabular}{ccc}
\hline Instrument & MJD start & MJD stop \\
\hline MAGIC & 58541.18 & 58547.21 \\
Fermi-LAT & 58540.72 & 58547.71 \\
NuSTAR & 58544.59 & 58545.31 \\
XMM & 58543.95 & 58544.21 \\
Swift-XRT & 58541.65 & 58560.71 \\
Swift-UVOT & 58541.65 & 58560.93 \\
KVA & 58541.22 & 58718.86 \\
Siena & 58542.17 & 58564.10 \\
\hline
\end{tabular}

In 3FGL and all prior Fermi-LAT catalogues the source had been indeed classified as a blazar candidate of uncertain type (Lefaucheur and Pita 2017) and only in 4FGL was it classified as a BL Lac object, with a photon index $\simeq 2$, which makes it an EHBL candidate (Abdollahi et al. 2020). EHBLs are of special interest in searching for new VHE gamma-ray blazars today as they are still rare and little studied (see e.g. Biteau et al. 2020). Some of them have shown a hard-TeV behaviour, with IC emission peaking above $\sim 10 \mathrm{TeV}$, while others were classified as EHBL showing extreme behaviour only during flares. According to Foffano et al. (2019) and Biteau et al. (2020), this may suggest the necessity for a classification of the EHBL class into different sub-classes.

Triggered by flaring activity in the high-energy gamma-ray band (HE, $0.5 \mathrm{MeV} \leq E \leq 100 \mathrm{GeV}$ ) reported by the Fermi-LAT (Cutini 2019), a MWL campaign on TXS 1515-273 was organised at the end of February 2019. During the flaring activity, the source was observed in different energy bands, ranging from radio to VHE gamma rays. Simultaneous or quasi-simultaneous observations were carried out by KVA, Swift, XMM, NuSTAR and MAGIC in order to investigate the location of the SED peaks and look for evidence of extreme behaviour during the flare. Following the observations MAGIC announced the first-time detection of the source in the VHE band (Mirzoyan 2019). In this paper, we report the results of this observing campaign.

The paper is organised as follows. In Section 2, we describe the analysis in the different energy ranges and provide the results obtained. In Section 3 we present the studies of the MWL variability. In Section 4 we report on the detailed analysis of the available X-ray datasets, focusing on the short-timescale variability and the spectral evolution. In Section 5 we describe the studies of the spectral energy distribution of TXS 1515-273, in particular its modelling and classification. Finally, in Section 6 we present our conclusions.

\section{ANALYSIS RESULTS}

Here we present a summary of the MWL analysis performed on the acquired data. The list of the instruments and the relative timerange of observations are provided in Table 1. Further details on each dataset are given in the following sections.

\subsection{Very-High-Energy gamma rays}

The Major Atmospheric Gamma-ray Imaging Cherenkov telescope (MAGIC) (Aleksić et al. 2016) is a stereoscopic system of two 17 $\mathrm{m}$ diameter imaging atmospheric Cherenkov telescopes. MAGIC telescopes performed observations of TXS 1515-273 starting on 27 February 2019 (MJD 58541) up to 05 March 2019 (MJD 58547).
The observations were performed at a high zenith angle, ranging from $55^{\circ}$ to $62^{\circ}$, under both dark time (4.8 hours) and moonlight conditions (3.3 hours), implying a high night sky background level. For this reason, we optimised the analysis chain for data taken under moonlight conditions. For further details on the MAGIC performance under moonlight, refer to Ahnen et al. (2017). Data were analysed using the MAGIC analysis and reconstruction software MARS (Zanin et al. 2013). The observations led to a significant detection in the VHE range with a significance of 7.6 $\sigma$. We derived the night-wise gamma-ray flux integrated above $400 \mathrm{GeV}$. The MAGIC observed flux is reported in Table A1.

Since the acquired signal was not strong enough to evaluate the spectrum for each night, we combined all the data to obtain the overall spectrum. We fitted it with a power-law function, folded with the energy dispersion using the Bertero unfolding method (Bertero 1989) and corrected for gamma-ray absorption by the interaction with the extra-galactic background light (EBL) using the Domínguez et al. (2011) model. After the unfolding and the EBL correction, the MAGIC soft spectrum between $200 \mathrm{GeV}$ and 900 $\mathrm{GeV}$ is well-described by a power-law model:

$\frac{d N}{d E}=N_{0}\left(\frac{E}{E_{0}}\right)^{-\Gamma}$,

where $\Gamma=3.11 \pm 0.32_{\text {stat }}$ is the photon index, $E_{0}=546 \mathrm{GeV}$ is the normalisation energy, selected as the decorrelation energy and $N_{0}=\left(1.76 \pm 0.28_{\text {stat }}\right) \times 10^{-11} \mathrm{TeV}^{-1} \cdot \mathrm{cm}^{-2} \cdot \mathrm{s}^{-1}$ is the corresponding normalisation constant. The systematic uncertainties of the MAGIC telescopes are: below $15 \%$ on the absolute energy scale, $11 \%-18 \%$ on flux normalisation and \pm 0.15 on the spectral slope (Aleksić et al. 2016). The soft spectrum in the VHE range suggests that the IC bump is likely peaking at $\mathrm{GeV}$ energies, as discussed in Section 5.3.

\subsection{High-Energy gamma rays}

The Large Area Telescope (LAT) instrument onboard the Fermi Gamma-Ray Space Telescope satellite is a pair-conversion telescope with a precision converter-tracker and calorimeter that detects gamma rays (Atwood et al. 2009).

Data from the Source event class were analysed using the Fermitools v1.2.1 and the fermipy ${ }^{1}$ v0.18 python package, applying standard quality cuts ('DATA_QUAL $>0$ \&\& LAT_CONFIG $==1$ ') and the zenith distance cut $\left(<90^{\circ}\right)$ to reduce the Earth limb contamination. Only events with reconstructed energy in the $300 \mathrm{MeV}$ - $500 \mathrm{GeV}$ range within $12^{\circ}$ of the nominal position of the studied source were selected. A time window of 7 days between 27 February 2019 (MJD 58541) and 05 March 2019 (MJD 58547) was selected in temporal coincidence with MAGIC observations to evaluate the measured flux as a function of the time. A different time selection, from 27 February 2019 up to and including 03 March 2019 (MJD 58545) was selected for the spectral analysis, for reason that will be explained in detail in Section 5.3. A binned likelihood analysis was performed with 8 bins per energy decade for the selected region of interest. The instrument response function used was P8R3_SOURCE_V2. All of the sources in the 4FGL catalog (Abdollahi et al. 2020) were included in the model, along with the isotropic (iso_P8R3_SOURCE_V2_v1) and the Galactic (gll_iem_v07) models (Acero et al. 2016) ${ }^{2}$.

\footnotetext{
${ }^{1}$ https://fermipy.readthedocs.io/en/latest/

2 https://fermi.gsfc.nasa.gov/ssc/data/analysis/software/
} 
In the fitting procedure the spectral parameters of sources that are significantly detected within a radius of $5^{\circ}$ around the source of interest were left free together with the normalisation of the diffuse components. All of the other catalog sources' parameters were fixed to the published 4FGL values. A dedicated likelihood analysis was performed for each time bin. The resulting light curve in daily bins is shown in Fig. 1, while the observed flux and the TS in each time bin are reported in Table A2.

The average spectrum obtained from Fermi-LAT data is described by a power-law model, with the following spectral parameters: $N_{0}=(20.5 \pm 7.2) \times 10^{-13} \mathrm{MeV}^{-1} \cdot \mathrm{cm}^{-2} \cdot \mathrm{s}^{-1}, \Gamma=2.2 \pm 0.3$ and $f_{\mathrm{E}>300 \mathrm{MeV}}=(4.5 \pm 1.3) \cdot 10^{-8} \mathrm{~cm}^{-2} \mathrm{~s}^{-1} \mathrm{GeV}$, with $E_{0}=2.3$ $\mathrm{GeV}$.

\subsection{X-rays}

Following the detection of the source at VHE gamma rays, TXS 1515-273 was observed at X-ray energies with the NuSTAR telescope, the XMM-Newton observatory and the Neil Gehrels Swift observatory. In this Section, we will present these observations and their outcomes.

\subsubsection{XMM-Newton observation}

The XMM-Newton observatory (Jansen et al. 2001) is a space-based $\mathrm{X}$-ray observatory which carries three medium spectral resolution X-ray telescopes, two Reflection Grating Spectrometers for high resolution spectroscopy (Den Herder et al. 2001) and a $0.3 \mathrm{~m}$ optical/UV imaging telescope on-board (see Section 2.4.2). The three X-ray telescopes at the focus of the European Photon Imaging Camera (EPIC, $0.2-10 \mathrm{keV}$ ) are a pn-CCD operating in full frame mode (Strüder et al. 2001) and two multi-object spectrometer CCDs (MOS1 and MOS2) operating in small-window mode (Turner et al. 2001).

The ToO XMM-Newton observation of TXS 1515-273 closest to our campaign was performed at the beginning of 02 March 2019 for $\sim 25 \mathrm{ks}$ of exposure time with the three X-ray telescopes. Using the XMM-Newton Science Analysis System SAS version 17.0.0 and the latest available calibration files, we reduced and analysed the data by following the standard procedure explained in the SAS user guide ${ }^{3}$. The source spectra were extracted from a source-centred circular region with a radius of 35 arcsec for all three instruments to have the best signal to noise ratio. The background spectra likewise were extracted from source-free regions on the same chips. We also used a time filter expression to divide the whole observation into short segments with durations of $300 \mathrm{sec}$. Then we extracted the final corresponding spectra from these segments to study the source variation over shorter time scales.

In order to estimate the Galactic column density of Hydrogen $\left(N_{H}\right)$ in the direction of the source, we used the full data set obtained by all three X-ray instruments on board of XMM-Newton. The spectra are binned in a way that each spectral bin contains 20 counts. The joint fit of the spectra is done using XSPEC v12.10.1s, using photon absorption model folded with a power-law and logparabola model, while the $N_{H}$ was set as a free parameter in our

aux/4fgl/Galactic_Diffuse_Emission_Model_for_the_4FGL_ Catalog_Analysis.pdf

3 https://xmm-tools.cosmos.esa.int/external/xmm_user_ support/documentation/sas_usg/USG/ analysis. Moreover, the model was multiplied by a constant to account for the cross-calibration between the three instruments. The multiplicative factor was fixed to 1 for the EPIC-pn and left as free parameter in the models which are used for MOS1 and MOS2. The observed spectrum can be described well by a power-law model with photon index $\Gamma_{\mathrm{XMM}}=2.53 \pm 0.01\left(\chi^{2} /\right.$ d.o.f.$\left.=2019 / 1882\right)$. We found that the log-parabola model cannot describe the observed spectrum better than the power-law model. The cross-calibration factor between the instruments was below $2 \%$. The value for the Galactic column density of Hydrogen from our spectral analysis is $N_{H}=(1.68 \pm 0.03) \times 10^{21} \mathrm{~cm}^{-2}$. The estimated value of $N_{H}$ is in agreement with the results presented in Willingale et al. (2013). Therefore, the Galactic column density of Hydrogen is fixed to the estimated $N_{H}$ value in the spectral analysis with shorter time bins and for the analysis of data from other X-ray instruments. The observed flux with XMM-Newton is reported in Table A3.

\subsubsection{NUSTAR observation}

The Nuclear Spectroscopic Telescope Array (NUSTAR) carries two co-aligned grazing incidence X-ray telescope systems, Focal Plane Module A and B (FPMA and FPMB), operating in a wide energy range of 3-79 keV (Harrison et al. 2013). These independent CdZnTe detector units provide X-ray imaging resolution of $18^{\prime \prime}$ (full width at half-maximum, FWHM) and spectral resolution of $400 \mathrm{eV}$ (FWHM) at $10 \mathrm{keV}$. The NUSTAR ToO observation of TXS 1515273 was performed at the end of 02 March $2019(\sim 9 \mathrm{~h}$ after the end of XMM-Newton observation) for a $34 \mathrm{ks}$ exposure time. The data reduction and product extraction were done using the NuSTAR Data Analysis Software NUSTARDAs v1.8.0 with a CALDB version 20180419. We performed the standard data reduction procedure explained in the NUSTAR user guide ${ }^{4}$. In order to extract the source spectrum, a source-centred circular region with a radius of 30 arcsec was used for both FPMA and FPMB. Likewise, the background was extracted from a source-free region with a larger radius of 60 arcsec. By creating user-selected good time intervals, we divided each exposure into 6 segments which enabled us to extract the spectra with durations of about 500-600 s each.

We fitted both spectra collected from FPMA and FPMB simultaneously in XSPEC v12.10.1s using a cross-calibration normalisation for each segment of observations. Due to the low count rate, we grouped all of the X-ray spectra to have at least 1 count per each energy bin. We then took the W-statistics (Wachter et al. 1979) into account to do the fitting procedure. The photon absorption model folded with a power-law is used by assuming a fixed $N_{H}$, which was estimated in Section 2.3.1. The cross-calibration factor was always below 3\%. The results of this analysis are presented in Section 4. The NuSTAR observed flux is reported in Table A4.

\subsubsection{Swift-XRT observations}

The Neil Gehrels Swift (Swift) satellite (Gehrels et al. 2004) carried out 8 observations of TXS 1515-273 between 30 September 2014 and 18 March 2019. The observations were performed with all three instruments on board: the X-ray Telescope (XRT; Burrows et al. 2005, 0.2-10.0 keV), the Ultraviolet/Optical Telescope (UVOT; Roming et al. 2005, 170-600 nm) (see Section 2.4.1), and the Burst Alert Telescope (BAT; Barthelmy et al. 2005, 15-150 $\mathrm{keV}$ ). The hard X-ray flux of this source turned out to be below

4 https://nustar.ssdc.asi.it/news.php\# 
the sensitivity of the BAT instrument for such short exposures and therefore the data from this instrument will not be used. Moreover, the source was not included in the Swift-BAT 70-month hard X-ray catalogue (Oh et al. 2018).

The multi-epoch event lists of the Swift-XRT data were downloaded from the publicly available Swift-XRT Instrument Log ${ }^{5}$. These observations were carried out in photon-counting mode. Following the standard Swift-XRT analysis procedure described by Evans et al. (2009), the data were processed using the configuration described by Fallah Ramazani et al. (2017) for blazars, assuming a photon absorption model folded with a power-law model and fixed $N_{H}$ as estimated in Section 2.3.1. In Table A5 we provide the results obtained from fitting the Swift-XRT spectrum.

Two Swift-XRT snapshots (MJD 58544.84 and 58544.97), simultaneous with the NUSTAR data, are combined with each other. A joint-fit was performed using these XRT data set and the full data obtained by NUSTAR. The observed spectra can be described by a photon absorption model (assuming fixed $N_{H}$ ) folded with a broken power-law model $\left(\chi^{2} /\right.$ d.o.f. $\left.=405 / 378\right)$. The spectrum shows a break at $3.34 \pm 0.34 \mathrm{keV}$. The photon indexes before and after the break energy are $\Gamma_{1}=2.10 \pm 0.11$ and $\Gamma_{2}=2.72 \pm 0.03$, respectively. The cross-calibration factor between Swift-XRT and NuSTAR data was $15 \%$.

\subsection{Optical and UV}

\subsubsection{Swift-UVOT observations}

During the Swift pointings, the UVOT instrument observed TXS 1515-273 in all of its optical $(v, b$ and $u)$ and UV ( $w 1$, $m 2$ and $w 2$ ) photometric bands (Poole et al. 2008; Breeveld et al. 2010). We analysed the data using the uvotsource task included in the HEAsoft package (v6.28) with the 20201026 release of the Swift/UVOTA CALDB. Source counts were extracted from a circular region of 5 arcsec radius centred on the source, while background counts were derived from a circular region of 20 arcsec radius in a nearby source-free region. The observed magnitudes are reported in Table A9.

The UVOT flux densities were corrected for Galactic extinction using the $\mathrm{E}(\mathrm{B}-\mathrm{V})$ value of 0.209 from Schlafly and Finkbeiner (2011) and the extinction laws from Cardelli et al. (1989). From the V-band fluxes, we subtracted the host galaxy contribution, which was estimated assuming a host galaxy absolute magnitude $M_{R}=-22.8$, galaxy colour V-R $=0.8$ (Fukugita et al. 1995), and $z=0.1285$. Within the aperture of five arcsec, its contribution is $0.13 \mathrm{mJy}$. We corrected only the V-band because the host galaxy contribution to other UVOT bands is negligible. We note that the magnitudes in Table A9 are the observed ones, i.e. corrections for neither the host galaxy contribution nor the Galactic extinction have been performed.

\subsubsection{XMM-OM observations}

The Optical Monitor (OM) observed the source in the $b, u$ and $w 1$ filters in imaging mode. The total exposure times of the imaging observations are approximately: $9400 \mathrm{~s}, 4700 \mathrm{~s}$, and $9400 \mathrm{~s}$. The data were processed using the SAS task omichain. The count rate is converted to flux using the conversion factors given in the SAS

5 https://heasarc.gsfc.nasa.gov/W3Browse/swift/ swiftxrlog.html watchout dedicated page ${ }^{6}$. The observed magnitudes with $X M M$ $O M$ are reported in Table A6. These magnitudes were then corrected for extinction using the same $\mathrm{E}(\mathrm{B}-\mathrm{V})$ value and extinction laws as for the UVOT data.

\subsubsection{KVA}

The optical R-band observations were performed using the $35 \mathrm{~cm}$ telescope attached to the $60 \mathrm{~cm}$ KVA telescope located at La Palma. The data analysis was performed using the standard procedures with the semi-automatic pipeline developed in Tuorla (Nilsson et al. 2018). As the source is not part of the Tuorla Blazar monitoring $\operatorname{program}^{7}$, a proper calibration was required in order to perform differential photometry. A comparison star and a control star were selected among known stars in the same field of view and were calibrated with respect to stars in the field of other targets observed on the same night under photometric conditions.

The magnitudes were corrected for Galactic extinction using the same $\mathrm{E}(\mathrm{B}-\mathrm{V})$ value as for correcting the UVOT data. The host galaxy flux was estimated in the same way as for the UVOT, resulting in a contribution of $0.303 \mathrm{mJy}$ within the used aperture of 5 arcseconds.

Observations of the source were performed for several months after the flaring state to estimate the flux during quiescent periods. The overall light curve is reported in Fig. 2, while the observed magnitudes (without host galaxy subtraction or Galactic extinction corrections) are reported in Table A8.

\subsubsection{Siena}

The Astronomical Observatory of the University of Siena observed TXS 1515-273 in the context of a program focused on optical photometry of blazars in support of MAGIC. The instrumentation consists of a remotely operated $30 \mathrm{~cm}$ Maksutov-Cassegrain telescope installed on a Comec 10 micron GM2000-QCI equatorial mount. The detector is a Sbig STL-6303 camera equipped with a 3072 x 2048 pixels KAF-6303E sensor; the filter wheel hosts a set of Johnson-Cousins BVRI filters. Multiple 300 s images of TXS 1515273 were acquired in the $\mathrm{R}$ band at each visit. Observations were always performed closely around culmination given the southern declination of the source. After standard dark current subtraction and flat-fielding, images for each visit were averaged and aperture photometry was performed on the average frame by means of the MaximDL software package. The choice of reference and control stars was consistent with the one for the KVA data. The obtained magnitudes reported in Table A7 were corrected for Galactic extinction and the host galaxy magnitude, which results in a contribution of $0.37 \mathrm{mJy}$ within the used aperture of 7 arcseconds, was subtracted as was done for the KVA data. Table A7 reports the magnitudes without these corrections.

\subsection{Very Long Baseline Array}

TXS 1515-273 has been observed six times with the Very Long Baseline Array (VLBA) Experiment (Lister et al. 2019) at $15 \mathrm{GHz}$ as part of the Monitoring Of Jets in Active galactic nuclei with

\footnotetext{
6 https://www. cosmos.esa.int/web/xmm-newton/ sas-watchout-uvflux

7 http://users.utu.fi/kani/1m/index.html
} 
VLBA Experiments (MOJAVE) program ${ }^{8}$. It was observed three times in 2017 (28 January, 25 May and 17 June), once in 2018 (31 May) and twice in 2019 (13 June and 19 July). We retrieve the fullycalibrated MOJAVE data sets and we produced images in Stokes' I, $\mathrm{Q}$, and $\mathrm{U}$ using the task IMAGR in AIPS. We then combined images in Stokes $\mathrm{Q}$ and $\mathrm{U}$ and produced polarisation intensity images and the associated error maps. We fitted the visibility data with circular Gaussian components at each epoch using the model-fit option in DIFMAP. Model-fitting the visibility reveals the core and two quasistationary components: the first one at 0.15-0.2 milliarcseconds from the core and the second one at 1.5 milliarcseconds (both with a nearly constant flux). From the data, there is no evidence for a new jet component emerging from the core, but the cadence of the observations is not optimal for detecting new components. The core flux was almost constant within the error bars, varying between $81 \pm 6$ (June 2019) and $99 \pm 7 \mathrm{mJy}$ (May 2017), in the first five epochs, while in the last epoch (July 2019) there is marginal hint of increased flux ( $109 \pm 8 \mathrm{mJy})$.

The source was not polarised in 2017 with an upper limit to the polarised flux density $\leq 0.2 \mathrm{mJy}$ and to the fractional polarisation $\leq 0.15 \%$. In 2018 May the polarised flux increased to $0.8 \pm 0.1 \mathrm{mJy}$, corresponding to a fractional polarisation of $0.5 \%$. In 2019 the polarised flux increased again to $1.5 \pm 0.2 \mathrm{mJy}$ (about $1.5 \%$ ) and $1.0 \pm 0.1 \mathrm{mJy}(0.8 \%)$ in June and July, respectively. However, the sparse time coverage does not allow us to set any robust connection between the polarised emission and the MWL activity.

\section{MULTI-WAVELENGTH VARIABILITY}

The light curves of TXS 1515-273 in the different wavelengths from radio to VHE gamma rays are reported in Fig. 1. The MWL light curves include all observations from MJD 58541 to MJD 58548. For comparison purposes, we show dashed grey lines in the FermiLAT and Swift-XRT light curves representing the reference value flux from the 4FGL catalog (Abdollahi et al. 2020) and a previous detection from Swift-XRT. From the reference level of the 4FGL catalog and a previous observation in the X-rays, it is evident that the source was in a high state in the X-ray and the HE band. The long term optical and HE gamma-ray light curves shown in Fig. 2 also indicate clearly that the observed flux in those energy ranges was higher than usual. A significant increase of activity was also observed by Swift-UVOT between 2014 and 2019 with all optical and UV filters (see Table A9).

The Fermi-LAT light curve shows a significantly higher flux in HE during the night of March 04, 2019, when the MAGIC observations in VHE gamma rays were prevented by bad weather. The Fermi-LAT SED was evaluated up to and including the night of March 03, 2019, to exclude this high state for which we did not have MAGIC data (see Fig. 1 and Section 5.3).

We also searched for intra-night variability in different bands. Intra-night variability was detected in X-ray observations on 02 March 2019 with both XMM-Newton and NuSTAR. The longest continuous MAGIC observations were $\sim 2$ hours, but due to lack of statistics we could not investigate further for intra-night variability. In the Fermi-LAT band the source is too weak to detect hour-scale variability as found in X-ray band. In optical band the variability in general has a rather small amplitude. We remark, however, that the XMM-Newton and NUSTAR observations, despite being very

8 https://www.physics.purdue.edu/MOJAVE/ close in time, were not overlapping: as a result, different variability timescales are not unexpected, as indeed was found (see Section 4). Consequently, we also decided to model the SED separately for the $X M M$-Newton and NuSTAR epochs, as we will discuss in detail in Section 5.3.

\section{SHORT-TIME X-RAY VARIABILITY}

Our observing campaign had particularly good coverage in X-rays, including XMM-Newton, Swift-XRT and NuSTAR. The long exposures of XMM-Newton and NUSTAR observations allow us to investigate in detail the short-time scale variability of the source in X-rays.

\subsection{Flare timescales}

The light curves showed multiple flares (see Fig.3 and 4) and in the following we investigate the details of this variability and use it to constrain physical parameters of the emission region.

To constrain the variability timescale, an exponential function in the form

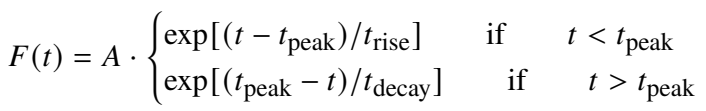

was used to fit the peak time, the rise and fall profiles of the bursts visible in the light curves. In both epochs, we fitted the soft and the hard energy band light curves independently, as shown in Figs 3 and 4. The results of the fit are reported in Table 2. We cross-checked the results of our fit with different time binning of the light curves, i.e. 5, 10 and 15 minutes for XMM-Newton and $\sim 10$ and $\sim 20$ minutes for NuSTAR. As a confirmation of the consistency of the results, no significant difference was found for the different binning.

We also tried several combinations for start and stop bins. First we selected a reasonable range for them to vary, performing the fit of the flare profile with different combinations of the range edges. We always kept the same fit range for soft and hard light curves. Finally, we picked the range that gave the best agreement with experimental data, i.e. with the minimum $\chi^{2}$ value. The parameter $t_{\text {peak }}$ was always set free to vary during the fitting procedure.

As shown in Figs 3 and 4, rapid variations on the time scale of the order of hours were detected in the two epochs. The derived timescales are not anomalous compared to X-ray flares seen in other blazars (MAGIC Collaboration et al. 2020a,b).

The flare profiles in all bands are symmetric, i.e. within the derived errorbars the rise and decay timescales of the flares are not significantly different. The rise times of both the observed X-ray flares are longer in the low-energy band than in the high-energy band. The difference is significant both in the XMM-Newton and NUSTAR results, with a confidence level higher than $99 \%$ and $90 \%$ for the two datasets respectively. In standard acceleration scenarios, low-energy electrons are supposed to be accelerated faster than high-energy ones. If the rise-time of the flare is dominated by the acceleration time-scale, then the rise-time in the lower energy band is expected to be shorter than the one in the high-energy band. However, higher energy electrons cool faster than lower energy electrons, and therefore in a cooling dominated regime one would indeed expect the higher energies to rise faster (e.g. Kirk et al. 1998) as seen in our data. This should also result in the high-energy flux peaking earlier than the lower energy flux, as seen in XMM-Newton data. In NUSTAR data this is not evident, which is in line with lower 


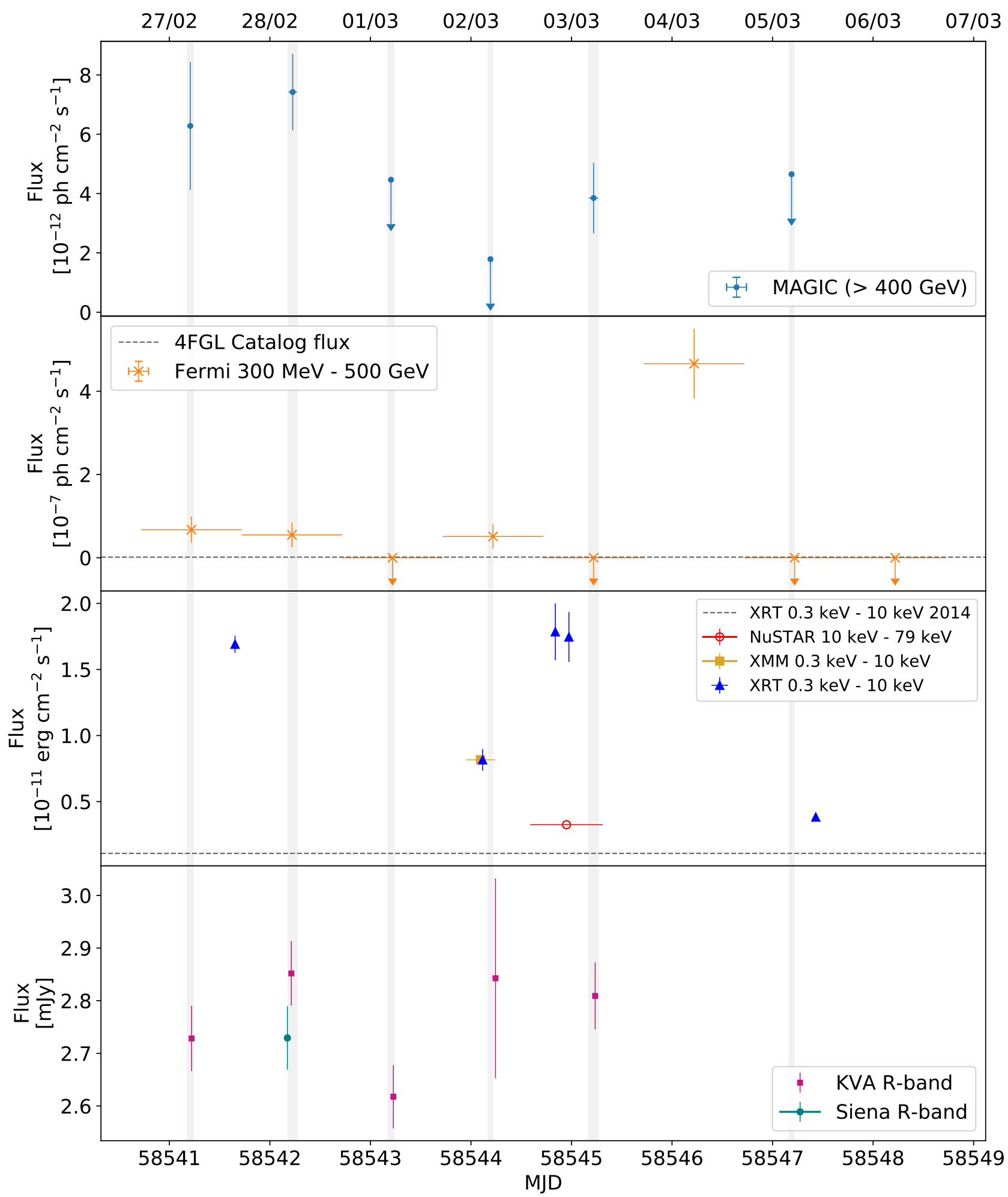

Figure 1. MWL light curve of TXS 1515-273 from 27 February 2019 to 06 March 2019. From top to bottom: VHE gamma rays by MAGIC, HE gamma rays by Fermi-LAT, X-rays by Swift-XRT (blue triangles), NuSTAR (red open circles) and XMM-Newton (yellow squares) in different energy bands and R-band by KVA and Siena observatory. 95\% confidence upper limits are indicated as downward arrows in VHE gamma rays where the flux is compatible with zero as well as in the HE band for each time bin where the test statistic (TS) value for the source was found to be smaller than 9. The individual light curves are daily binned. Dashed horizontal grey lines indicate the level of detected flux during 2014 Swift-XRT observations and the reference flux level in the 4FGL catalog. The grey vertical bands are drawn to highlight the MAGIC observation time slots. Magnitudes for optical data were corrected for Galactic extinction, and the host galaxy contribution was subtracted (details in the text). 


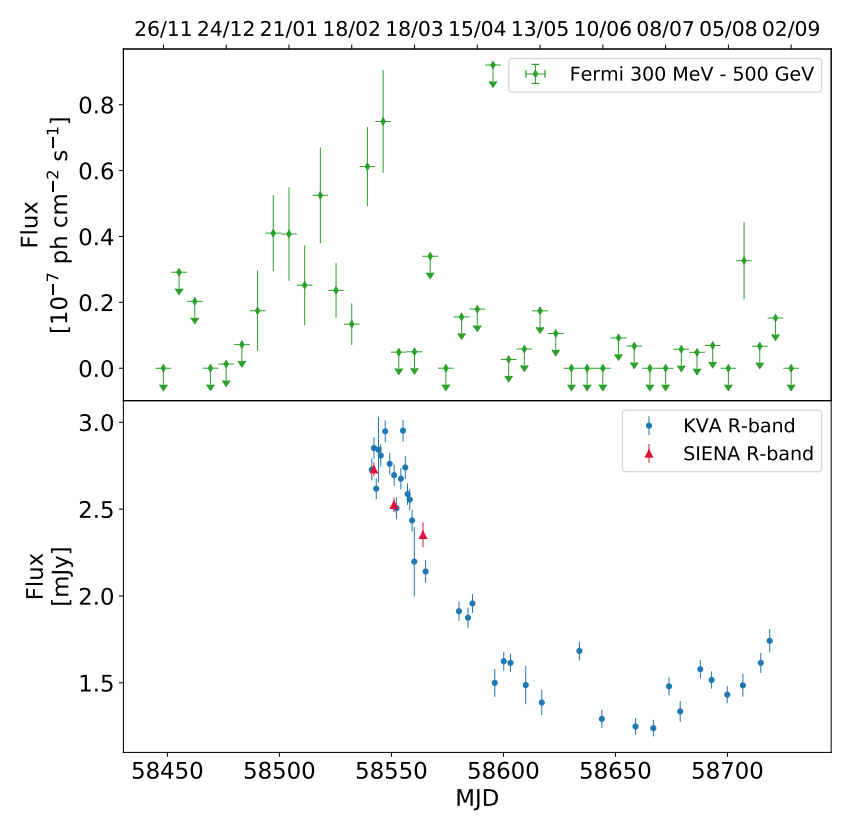

Figure 2. HE gamma-ray (top, green diamonds) and R-band (bottom, KVA in blue circles and Siena observatory in red triangles) light curves of TXS 1515-273. Fermi-LAT light curve is shown in weekly bins, with $95 \%$ confidence UL indicated. The light curve starts from a few months before the flare period up to August 2019. The large flare can be seen during our observations. The optical light curve has daily bins, starting from the observations carried out during the flare.

Table 2. Results of the fit of the burst profile for the XMM-Newton and the NUSTAR epoch. The starting time corresponds to MJD 58543.0 for XMMNewton and to MJD 58544.6 for NUSTAR.

\begin{tabular}{ccccc}
\hline Instrument and energy & $\begin{array}{c}t_{\text {rise }} \\
(\min )\end{array}$ & $\begin{array}{c}t_{\text {peak }} \\
(\mathrm{min})\end{array}$ & $\begin{array}{c}t_{\text {decay }} \\
(\mathrm{min})\end{array}$ & $\begin{array}{c}t_{\text {peak }} \\
(\mathrm{MJD})\end{array}$ \\
\hline$X M M(0.3-3 \mathrm{keV})$ & $316 \pm 34$ & $107 \pm 3$ & $262 \pm 32$ & 58544.06 \\
$X M M(3-10 \mathrm{keV})$ & $159 \pm 29$ & $93 \pm 6$ & $193 \pm 37$ & 58544.06 \\
NuSTAR $(3-10 \mathrm{keV})$ & $225 \pm 42$ & $773 \pm 10$ & $123 \pm 17$ & 58545.14 \\
NuSTAR $(10-79 \mathrm{keV})$ & $127 \pm 41$ & $771 \pm 16$ & $86 \pm 18$ & 58545.14 \\
\hline
\end{tabular}

statistical significance in difference of rise times between the two bands as well.

\subsection{Spectral evolution}

We investigated the dependence of the photon index on the flux in the full energy range $(0.3-10 \mathrm{keV}$ for XMM-Newton, $3-79$ $\mathrm{keV}$ for NuSTAR). The XMM-Newton photon index showed little variations, ranging between $2.35 \pm 0.05$ and $2.76 \pm 0.06$ (using 5minute binning within the full time range of observation), while the NUSTAR photon index showed a wider dynamical range, varying between $2.01 \pm 0.26$ and $4.33 \pm 0.71$ (using 10-minute binning within the full time range of observation). In both of the epochs studied, this dependency showed a more complicated behaviour than simple spectral hardening. We also examined the relation between

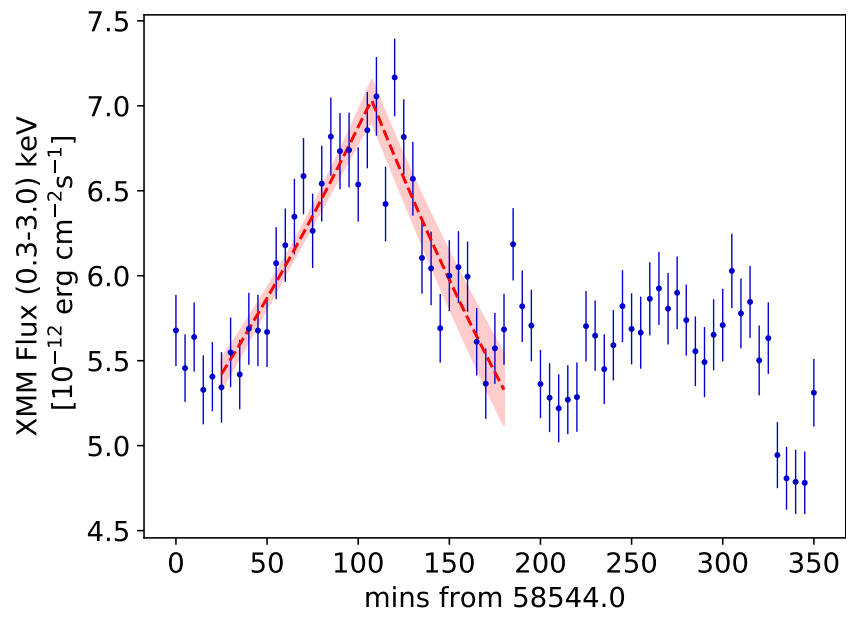

(a) XMM-Newton 0.3-3 keV 5-minute bins light curve for TXS 1515-273.

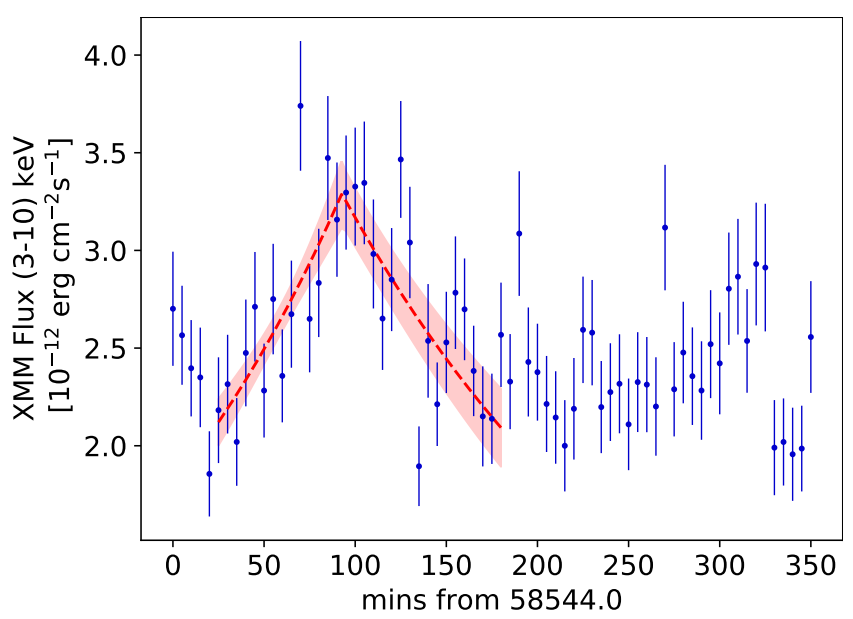

(b) XMM-Newton 3-10 keV 5-minute bins light curve for TXS 1515-273.

Figure 3. XMM-Newton light curves for TXS 1515-273 for soft (top) and hard (bottom) energy band. The dashed curves superimposed on the data represents the exponential function used to fit the peak, the rise and decay time of the bursts. The red shaded areas represent the uncertainties of the fit.

the integral flux and the hardness ratio, but no clear correlation between these quantities was found in our data sample.

We then focused our search on the most prominent flares in the X-ray light curves (i.e. the one we also used to constrain the variability time scale), see Fig. 5 . We slightly narrowed the time window with respect to the time range shown in red in Figs. 3 and 4 focusing on the most prominent flare time slots, MJD 58544.00 58544.08 , (i.e. 25 - 125 minutes after the beginning of the observations) for XMM-Newton and MJD 58545.08 - 58545.17, (i.e. 670 810 minutes after the beginning of the observations) for NuSTAR. In this time epoch, the data are statistically consistent with a simple harder-when-brighter trend. Indeed, we fitted the data in both epochs with a linear function and we found that the reduced $\chi^{2}$ is 0.97 for the XMM-Newton flare and 0.55 for the NuSTAR flare.

\subsection{Constraints on emitting region parameters from variability timescales}

Using the shortest time scale from these variability studies for individual epochs, the size of the emission region can be constrained 


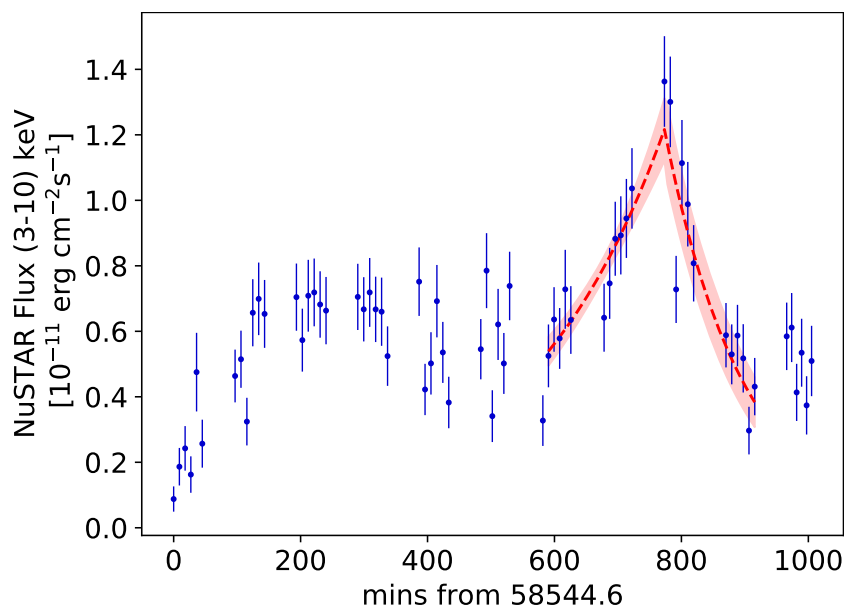

(a) NUSTAR 3-10 keV 10-minute bins light curve for TXS 1515-273.

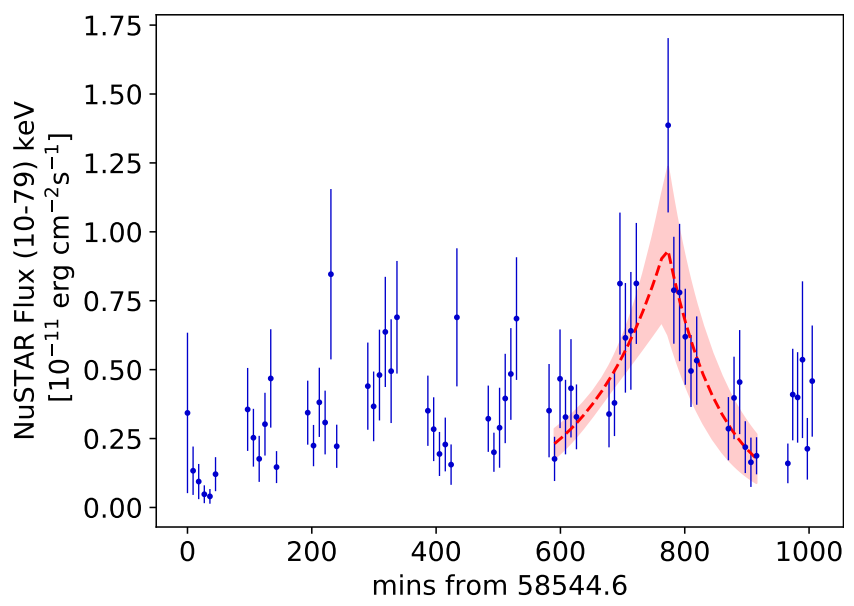

(b) NuSTAR 10 - $79 \mathrm{keV}$ 10-minute bins light curve for TXS 1515-273.

Figure 4. NUSTAR light curves for TXS 1515-273 for soft (top) and hard (bottom) energy band. The dashed curves superimposed on the data represents the exponential function used to fit the peak, the rise and decay time of the bursts. The red shaded areas represent the uncertainties of the fit.

as a function of the Doppler factor $\delta$ :

$R \leq \frac{c t_{\mathrm{var}} \delta}{(1+z)}$,

where we used $t_{\mathrm{var}}=159 \pm 29 \mathrm{~min}$ for the XMM-Newton epoch and $t_{\mathrm{var}}=86 \pm 18 \mathrm{~min}$ for the NuSTAR epoch (see Table 2).

Since we have no strong constraints on the Doppler factor neither from our observing campaign nor from the previous observations of the source, we used $\delta \sim 20$ first, but then iterated the value during the SED modelling (see Section 5.3). The values close to 20 are rather typical for VHE gamma-ray emitting BL Lacs (Tavecchio et al. 2010).

As we were able to constrain the variability timescales in both epochs in the two different energy bands, we followed Zhang et al. (2002) to use these timescales to constrain the magnetic field strength of the emission region. The synchrotron cooling time of electrons is inversely proportional to the square root of the energy of the photons. Indeed, the results of our fit are consistent with this scenario, as we find the XMM-Newton lower energy photons cooling time is longer than the one found for the NUSTAR higher energy ones.

The magnetic field strength is then calculated using the formula

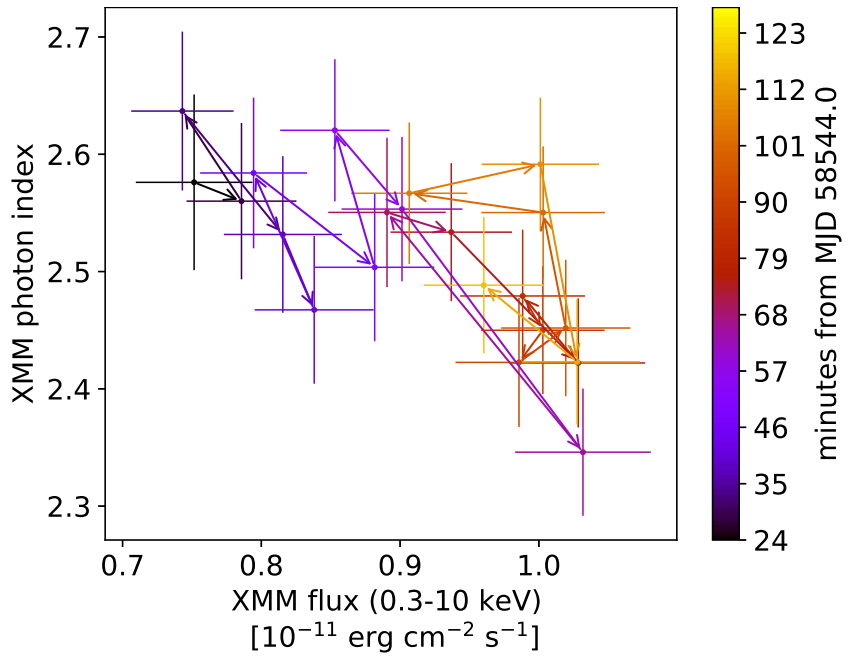

(a) XMM-Newton photon index versus integral $(0.3-10 \mathrm{keV})$ flux for TXS 1515-273 during the short flare observed (MJD 58544.00 - 58544.08, i.e. 25 - 125 minutes after the beginning of the observations).

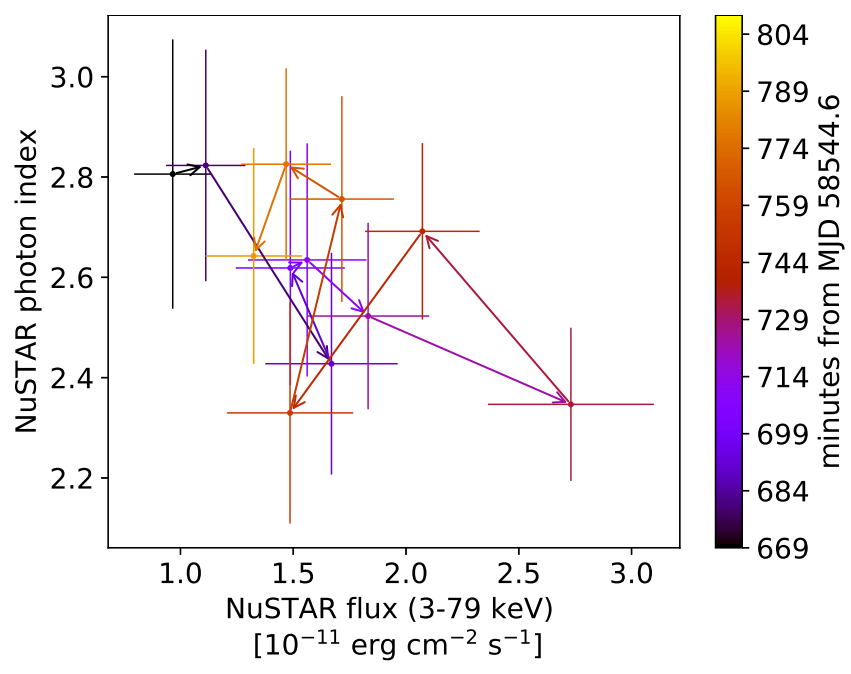

(b) NUSTAR photon index versus integral $(3-79 \mathrm{keV})$ flux for TXS $1515-$ 273 during the short flare observed (MJD 58545.08 - 58545.17, i.e. 670 810 minutes after the beginning of the observations).

Figure 5. XMM-Newton (top) and NuSTAR (bottom) photon index versus integral flux for TXS 1515-273 during short X-ray flares. The coloured arrows represent the time evolution.

from Zhang et al. (2002):

$B=210 \times\left(\frac{1+z}{E_{l} \times \delta}\right)^{1 / 3}\left[\frac{1-\left(E_{l} / E_{h}\right)^{1 / 2}}{\tau_{\text {soft }}}\right]^{2 / 3} \quad \mathrm{G}$

where $E_{l}$ and $E_{h}$ are taken as the logarithmic mean energies in the low and high-energy band in units of $\mathrm{keV}$ for the low-energy band and the high-energy band considered, and $\tau_{\text {soft }}$ is the difference in the decay time values for the energy bands considered. We decided to combine the observations from the two epochs because the time lag in our observations is not statistically significant. By working under the assumption that the magnetic field does not vary between the two epochs, we used the low-energy band of XMM-Newton and the highenergy band of NuSTAR, and $\tau_{\text {soft }}=t_{\text {decay,XMM }}-t_{\text {decay,NuSTAR }}=$ $10.56 \pm 2.20 \mathrm{ks}$

The upper limit value of $R$ and the estimated value of $B$ cor- 
Table 3. Results of the variability time-scale fit and estimation of $R$ and $B$.

\begin{tabular}{cccc}
\hline Epoch & $\delta$ & $\begin{array}{c}R \\
\left(\times 10^{15} \mathrm{~cm}\right)\end{array}$ & $\begin{array}{c}B \\
(\mathrm{G})\end{array}$ \\
\hline XMM & 20 & $5.07 \pm 0.92$ & $0.14 \pm 0.02$ \\
NuSTAR & 20 & $2.73 \pm 0.58$ & \\
\hline
\end{tabular}

responding to $\delta=20$ obtained with the variability timescales estimated from the two epochs are reported in Table 3. These values were used to model the SED, as we will describe in Section 5.3.

\section{SPECTRAL ENERGY DISTRIBUTION}

In this campaign, we measured the SED of TXS 1515-273 for the first time from the optical to the VHE gamma-ray band. This allowed us to investigate the source classification and possible emission models during the flaring state.

\subsection{Source classification}

As reported in Biteau et al. (2020), it is possible to distinguish three kind of extreme behaviours: HBL sources showing extreme behaviour during flaring states, when synchrotron and IC peaks shift towards higher frequencies, going back to their standard HBLlike state, as observed for Mrk501 (Ghisellini 1999); sources with a steady hard synchrotron without evidence for a hard spectrum at $\mathrm{TeV}$ energies; finally, sources showing a persistently hard IC hump peaking at and above $\mathrm{TeV}$ energies and a synchrotron peak in the $\mathrm{X}$-ray band. Our excellent X-ray and VHE gamma-ray data allowed us to search for these signatures in the SEDs that we measured at different times for TXS 1515-273.

TXS 1515-273 was very little studied before the flare occurred in 2019. As explained in the previous Sections, the photon index reported by Abdollahi et al. (2020) seemed to suggest a possible EHBL classification. Thus, we examined archival data from the ASI Space Science Data Center (SSDC) ${ }^{9}$ to test if the source could indeed be classified as such. According to Bonnoli et al. (2015), a good criterion to select EHBLs relies on the high X-ray-to-radio flux ratio. As reported by the authors, a high ratio of $\mathrm{X}$-ray versus radio flux $\left(F_{X} / F_{R}>10^{4}\right)$ would indicate a good EHBL candidate. Based on archival data, considering the flux in the $\sim 10^{16}-10^{19} \mathrm{~Hz}$ band and in the $\sim 10^{9}-10^{10} \mathrm{~Hz}$ band for $F_{X}$ and $F_{R}$ respectively, we found a ratio of $\sim 30$, which led us to the conclusion that it was not a good EHBL candidate.

In order to estimate the peak frequency and classify the source, we combined strictly simultaneous data in the optical and X-ray band to perform a fit of a log-parabola to the data in the log-log plane:

$v F_{v}(v)=f_{0} \cdot 10^{-b \cdot\left(\log _{10}\left(v / v_{\mathrm{s}}\right)\right)^{2}}$,

where $v_{\mathrm{S}}$ is the peak frequency. This fit was performed separately on data from the current observations and a previous Swift-UVOT and Swift-XRT observations from 2014. We considered data from the two epochs separately in order to take into account a possible shift in the peak frequency between the two observing periods, combining Swift-XRT observations from MJD 58544.84 and 58544.97 with the NUSTAR dataset. The results of the fit procedure on the three

9 http://www.asdc.asi.it/
Table 4. Results of the log-parabola fit of the synchrotron peak of the SED of the three different epochs considered.

\begin{tabular}{cc}
\hline Epoch & Log of peak frequency $(\mathrm{Hz})$ \\
\hline 2014 Swift & $13.46 \pm 2.53$ \\
XMM & $15.28 \pm 0.06$ \\
NuSTAR and 2019 Swift & $15.56 \pm 0.11$ \\
\hline
\end{tabular}

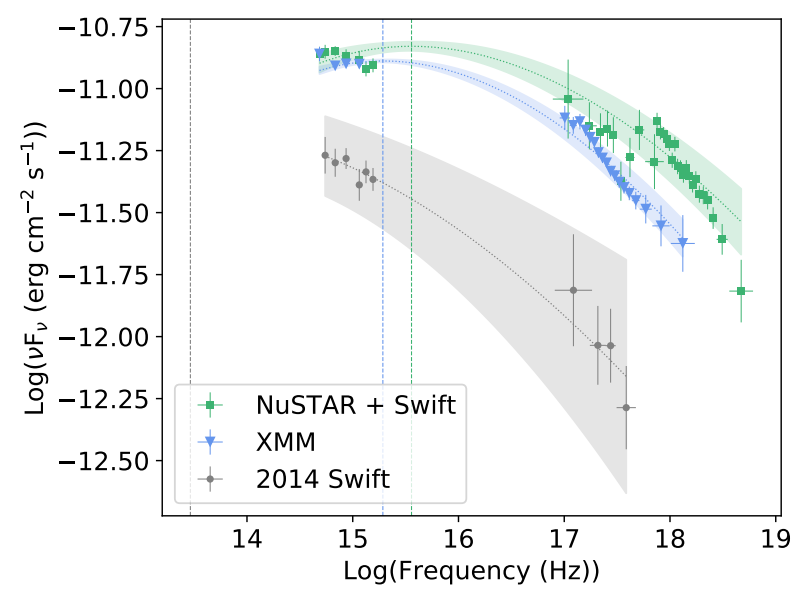

Figure 6. Results of the fit of the SED synchrotron bump with a log-parabola. Data are shown in different colours, representing: NuSTAR epoch (NuSTAR and Swift datasets taken on MJD 58544.84 and 58544.97

, green squares), XMM epoch (XMM-Newton and XMM-OM dataset, blue triangles), 2014 Swift dataset (grey circles). Dotted curves are showed superimposed on SED points with the same colours, representing the fit curves and the uncertainty bands. Vertical dashed line are also shown, in correspondence of the peak frequency evaluated from the fit parameters.

considered datasets are reported in Fig. 6. The best fit values for the three datasets are reported in Table 4.

The best fit values found for the most recent observations classify the source as HSP during the flare. For 2014 data, however, the value found $v_{\mathrm{S}} \simeq 10^{13.46 \pm 2.53} \mathrm{~Hz}$ does not allow any conclusion to be drawn on the classification of the source. However, it is clear that $v_{\mathrm{S}}$ moved to higher energy between the XMM-Newton and the NUSTAR observations, which were separated by less than a day. This is most naturally explained by the injection of fresh electrons or the dominance of a new emission component. Even in the flaring state detected by NUSTAR the peak frequency does not exceed $10^{17}$ and therefore it is clear that TXS 1515-273 is not an extreme synchrotron peaked source.

\subsection{Emission models}

Most blazars' observations are fitted with radiative models. These models are usually classified as leptonic and hadronic models.

In the simple one-zone SSC model, TeV emission is the result of the IC scattering of electrons and positrons in the jet on photons created by the electron population itself via synchrotron emission. The SSC model is supported by several observations of good temporal correlation between the $\mathrm{TeV}$ and X-ray flares (see Maraschi et al. 1999; Takahashi et al. 2000; Krawczynski et al. 2001; Coppi and Aharonian 1999). In this simple one-zone SSC 
model the energy density has been found to be largely dominated by particle energy density rather than the magnetic field; this unusually low magnetisation seems to be in contradiction with theoretical and observational constraints of equipartition conditions, which cannot be reproduced in BL Lac objects with the one-zone model (Tavecchio and Ghisellini 2016). It was recently suggested, however, that equipartition can be achieved in one-zone models via the introduction of an anisotropic electron population (Tavecchio and Sobacchi 2020). The one-zone SSC is still the default model and, given the fact that during this campaign all of the energy bands were found to be in the same high state, we apply this model to the data using the constraints for the size of the emission region and magnetic field strength we found in Section 4.3.

Another possible way to solve the contradiction on the low magnetization can be obtained by taking into consideration the existence of additional seed photons from other parts of the jet (e.g. Georganopoulos and Kazanas 2003; Ghisellini et al. 2005). In these models one assumes the jet to be structured. As shown in Tavecchio and Ghisellini (2015), the assumption of a supplementary source of soft photons intervening in the IC emission allows the reproduction of the observed SED assuming equipartition between the magnetic and the electron energy densities. There is also observational evidence for spine-sheath models from very long baseline interferometry observations (e.g. Attridge et al. 1999; Giroletti et al. 2004) and indications for multiple components contributing to the optical band from long-term variability (Lindfors et al. 2016) and polarization (see e.g. Valtaoja et al. 1991).

In our case, the X-ray variability indicates that the X-ray emission region is very compact. Therefore, it must either be located very close to the central engine (to fill the full diameter of the conical jet) or be embedded in a larger emission region. Our X-ray light curves show several flares and the SED peak moves to higher energies from the XMM-Newton epoch to the NUSTAR epoch. This can be either due to the variation of the particle distributions within the emission region (e.g. fresh injection of particles) or due to the flaring region (filling the full diameter of the jet) consisting of several emission regions. Therefore, we also model the SEDs with a two-component model such that we keep the parameters of the larger region constant between the two SED epochs and only vary the parameters of the small emission region.

Another possible explanation of the VHE emission observed is given by hadronic models (e.g. Mannheim 1993; Mücke and Protheroe 2001; Aharonian and Taylor 2010; Cerruti et al. 2015), where energetic photons are produced in jets via hadronic interactions. These models are based on the assumption of the presence of high-energy protons in the jet which are accelerated together with the electrons. While the low-energy peak is still explained by synchrotron radiation of electrons, in this scenario the VHE gamma rays are thought to be produced by interactions of the relativistic protons with soft photons or with the magnetic field. In particular, the recent indication of a link between a neutrino track event and the blazar TXS 0506+056 (Aartsen et al. 2018a,b) can be interpreted in a lepto-hadronic scenario where electrons and protons are accelerated in the jet, and synchrotron photons from the electrons lead to photo-hadronic neutrino production (Keivani et al. 2018; Ansoldi et al. 2018; Cerruti et al. 2019).

However, since our study shows that TXS 1515-273 is a rather typical HSP in flaring state and since no neutrinos have been detected from the direction of TXS 1515-273 (Aartsen et al. 2020), in the next Section we investigate only one-zone and two-component leptonic models for the spectral energy distribution.

\subsection{Spectral energy distribution modelling}

We derived the broadband SED from radio to VHE shown in Figs 7 and 8. For comparison purposes, archival data from the SSDC and from a previous Swift-XRT detection are also shown as grey dots.

The light curve in the Fermi-LAT band showed the presence of a high flux state on MJD 58546. Since no VHE observations were available on that day, we performed the Fermi-LAT spectral analysis starting from MJD 58541 up to MJD 58545 in order to have a smooth connection between the HE and the VHE gamma-ray observations. For what concerns the optical and $\mathrm{X}$-ray observations, since these were not simultaneous and the source showed short-term variability in the X-ray energy range, we decided to separate the datasets into NuSTAR, Swift-XRT and Swift-UVOT observations and XMMNewton and XMM-OM observations. We modelled these datasets separately since the model we adopted is not time-dependent.

Given the fact that the source was in a high state in all energy bands, the SED was at first modelled with a simple one-zone SSC model; the radiation is emitted in a region in the jet by a single homogeneous population of electrons (Maraschi and Tavecchio 2003) which is responsible for the emission from infrared to VHE frequencies. The emitting region can be described as a sphere with radius $R$ with a uniform magnetic field $B$. The Doppler factor, $\delta$, is required to take into account the relativistic effects. For HSP sources, this factor is usually $\sim 10-50$ : within this range we selected a value for each epoch that would reproduce a model in good agreement with our data, having a consistent model by using the same value of $\delta$ to estimate $R$ and $B$.

A good agreement between the model and the SED data was found using $\delta=18$ for the XMM-Newton epoch, which corresponds to an estimated magnetic field intensity $B=0.14 \pm 0.02 \mathrm{G}$ and to an upper-limit on the size equal to $R \leq 4.56 \pm 0.83 \times 10^{15} \mathrm{~cm}$, and $\delta=$ 24 for the NuSTAR epoch, which corresponds to $B=0.13 \pm 0.02 \mathrm{G}$ and $R \leq 3.28 \pm 0.69 \times 10^{15} \mathrm{~cm}$.

The population of relativistic electrons is described by a broken power-law model, where $K$ is a normalisation factor which represents the density of electrons with $\gamma=1, n_{1}$ the index from $\gamma_{\min }$ to $\gamma_{\mathrm{br}}$ and $n_{2}$ from $\gamma_{\mathrm{br}}$ to $\gamma_{\max }$, for a total of six parameters. The model includes the Klein-Nishina cross section $\sigma_{K N}$ for the IC spectrum calculation, which is important in the case of emission above the GeV range. Since our analyses in Sections 4.2 and 5.1 clearly demonstrated that between the XMM-Newton and NuSTAR observing epochs the synchrotron peak moved to higher energies and spectral evolution with time changed the pattern, we assume that the new blob dominates the emission in the NUSTAR epoch, i.e. we let the electron energy spectrum parameters vary rather arbitrarily between the two epochs.

For both datasets, we found acceptable agreement between the models and the data available from optical to VHE gamma-ray (see Figs. 7a and 8a). The parameters of the one-zone model are given in Table 5. Even if we tried a large number of different combinations of parameters, it was hard to find parameters that would reproduce well the shape of the synchrotron bump. Parameters that would describe well the shape of the spectrum in the X-ray band led to an underestimation of the optical flux while changing the set of parameters so that the optical shape was well described by the model would overproduce the X-ray flux. Moreover, we also had to take into account the IC bump which extends to VHE gamma-ray energies. In the XMM-Newton epoch, the biggest challenge for the model is to reproduce the rather soft XMM-Newton spectra and still let the second peak extend to VHE gamma-rays without overproducing the HE gamma-ray part. The selected model is a compromise and 
is below the VHE gamma-ray points. In the NUSTAR epoch, the selected model produces well the level of the flux in the UV-band and the shape and level of the NUSTAR observations, but does not describe the shape in the optical band and overproduces the SwiftXRT data. The second bump is well described in the NuSTAR epoch.

As expected, the one-zone model is not able to reproduce the emission at radio frequencies $(15 \mathrm{GHz})$ : this is due to the small and dense emission region. The emission foreseen by the one-zone model is synchrotron self-absorbed. It is likely that the radio flux originates from a different, larger, region of the jet, transparent at those frequencies. Also, as is typical for one-zone models, both the $X M M$-Newton and NuSTAR one-zone models are far from equipartition (see Table 5, last column).

The two-component model could potentially improve the modelling of the synchrotron component and there exists numerous observational evidence supporting two-component models for BL Lac objects in general (see Section 5.2). Therefore we modelled the observed SEDs with the two-component model described in MAGIC Collaboration et al. (2020c), and references therein. The model includes two spherical blobs embedded in one another which are interacting. Each of the blobs is filled with relativistic electrons with a broken power-law distribution. The two blobs have the same magnetic field strength, but different Doppler factors and sizes, increasing the number of parameters from eight to fifteen. We call the two emission regions "blob" (smaller region) and "jet" (larger region). The interaction between the emission regions provides additional seed photons for Compton scattering. The larger emission region dominantly provides seed photons to the smaller region (Tavecchio et al. 2011). The gamma-gamma absorption is negligible (see MAGIC Collaboration et al. 2020c). The two-component model describes the data from the radio to the VHE range well (see Figs. $7 \mathrm{~b}$ and $8 \mathrm{~b}$ ) with the parameters given in Table 5. The improvement with respect to the one-zone model is most evident in the radio and optical part. While the lowest radio frequencies are still not reproduced in the two-component model, the "jet" component connects smoothly the $15 \mathrm{GHz}$ MOJAVE point to the optical data and the shape of the optical SED is reproduced better. The jet component is closer to equipartition, but we do not find solutions where the blob component itself would be in equipartition; indeed, for $X M M$ Newton and NuSTAR we find values of the order $10^{-3}-10^{-4}$. The main difficulty is to produce the Compton dominance we see in the observed SED.

\section{SUMMARY AND DISCUSSION}

In this paper we present a MWL analysis of the source TXS 1515273, a BL Lac object which had been very little studied before this campaign. When compared to archival data in the X-ray and HE gamma-ray energy bands and optical monitoring following the analysed period, at the end of February 2019 the source was found to be in a flaring state in all energy bands, which lead to its first detection in the VHE gamma-ray band by MAGIC. Since this time, no further $\mathrm{TeV}$ observations have been published so no additional information on its VHE gamma-ray emission is available.

Thanks to the very good X-ray coverage of the source during the flaring state it was possible to detect an hour-scale variability in the X-ray fluxes and a clear change in the spectral shape between the XMM-Newton (MJD 58544) and NuSTAR (MJD 58545) observations. We produced the XMM-Newton and NUSTAR light curves in two energy bins each and constrained the rise and decay times of the flares. We find rapid variations on the time scale of the order of hours in both datasets. Studies of variability of blazars in the NUSTAR band (Bhatta et al. 2018) show that the timescale we found is in line with the fastest variability seen in many HSP sources. In few cases, even shorter timescales variability has been found, e.g., for Mrk 421 (MAGIC Collaboration et al. 2020a) and for 1ES 1959+650 (MAGIC Collaboration et al. 2020b), although different analytical functions were adopted in these works to profile the flares. The short time-scale variability was used to constrain the size of the emission region and the intensity of the magnetic field, both used later during the analysis to find a suitable model for the broadband emission.

TXS 1515-273 was very little studied prior to this work and therefore the source had not yet been properly classified. Up to and including the 3FGL Fermi-LAT catalog, the source was still classified as a blazar candidate of uncertain type. Only in the 4FGL catalog was it classified as a BL Lac object, but the SED type was not defined. The good coverage in the optical-X-ray energy band obtained in this observational campaign allowed an accurate determination of the synchrotron peak frequency $v_{\mathrm{S}}$ and the classification of the source as a HSP source during this flare. Furthermore, during the flare, the peak clearly shifted to higher energies in a timescale of less than a day. Such behaviour is rather common in blazars, see e.g. Pian et al. (1998) or MAGIC Collaboration et al. (2020b), in which a clear shift in the synchrotron bump was reported during flare activity with respect to archival data. In our case, thanks to very good X-ray coverage we could follow this shift on daily time scales.

We also investigated the behaviour of the jet at $15 \mathrm{GHz}$ using the VLBA data from the MOJAVE program. We found no new components or moving components, which is in line with what is seen in other $\mathrm{TeV}$ blazars with high synchrotron peak frequency (see e.g. Piner and Edwards 2018, and references there in). HSP objects usually do not show high linear core polarization levels (Lister et al. 2011), which is what we also find for TXS 1515-273 in the first epochs of data. However, in 2019 the core was significantly polarized, which might be connected to the general high state, but the sparse coverage of the VLBA observations did not allow a strong conclusion to be drawn.

We also modelled the broadband SED from radio to VHE gamma rays. The evolution of the SED from the XMM-Newton to the NUSTAR epoch clearly suggests that an injection of new electrons or a new blob is starting to dominate the emission. The one-zone model describes well the data from the X-ray band to VHE gamma rays for both epochs, but has problems reproducing the shape of the optical part of the SED and does not reproduce the radio. The latter is expected as the small emitting region considered is optically thick to radio emission. The radio emission must then originate from a different component. These issues are solved when a twocomponent model is considered, in which the blob is responsible for the emission from X-ray to VHE gamma rays, while the jet dominates the emission at radio wavelengths. The introduction of the two-component model increases the number of model parameters from eight to fifteen, allowing for a more accurate modelling of the emission. In particular, the jet emission results in important contributions in the optical band. Moreover, assuming the two emission regions to be co-spatial, seed photons for the inverse Compton scattering are provided also from the jet.

Finally, we examined the ratio between the magnetic field density and the electron energy density, $U_{\mathrm{B}} / U_{\mathrm{E}}$. In both epochs, we find that the one-zone SED parameters are quite far from equipartition, with the magnetic field energy density dominated by the kinetic energy of the relativistic particles by several orders of magnitude. 


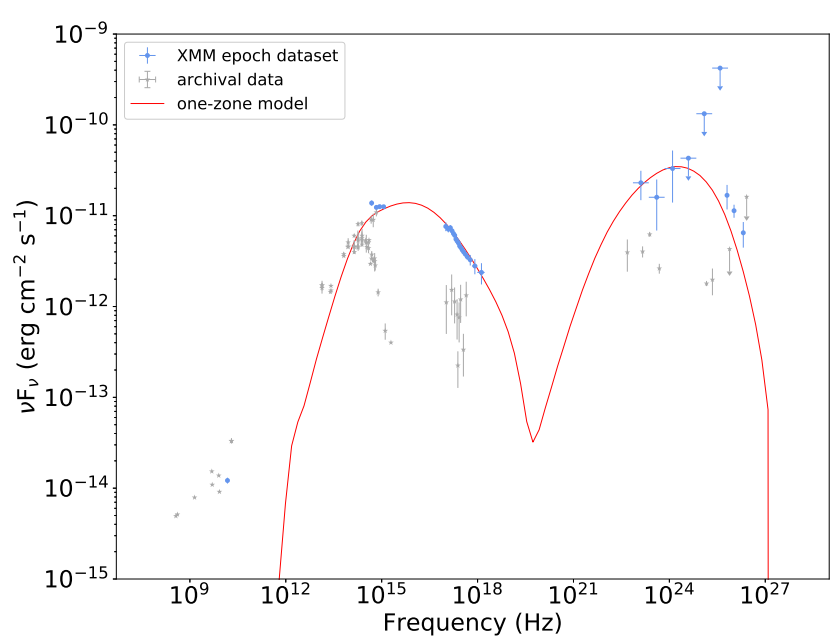

(a)

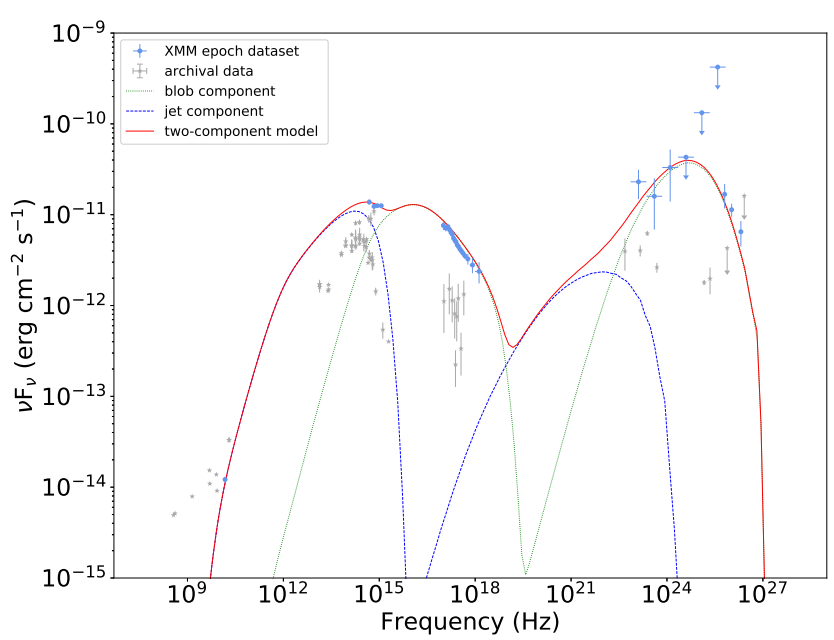

(b)

Figure 7. Broadband SED and modelled spectra for TXS 1515-273. The SED has been modelled considering the VLBA SED point in the radio band, KVA, $X M M-O M$ and XMM-Newton SED points in the optical and X-ray energy band and the Fermi-LAT and MAGIC SED points in the gamma-ray band

, using a one-zone model (left) and a two-component model (right). Coloured points represent observations in the different energy bands during the observations of 2019, grey points represent archival data. The red solid line represents the models, the green dotted line represents the blob emission and the blue dashed line represents the jet emission. Further details can be found in the text. See Table 5 for parameters.

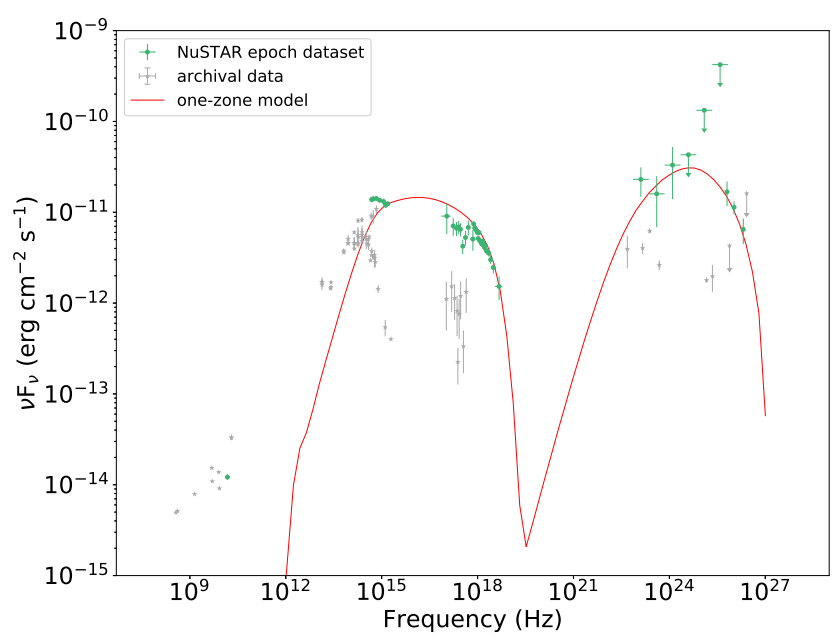

(a)

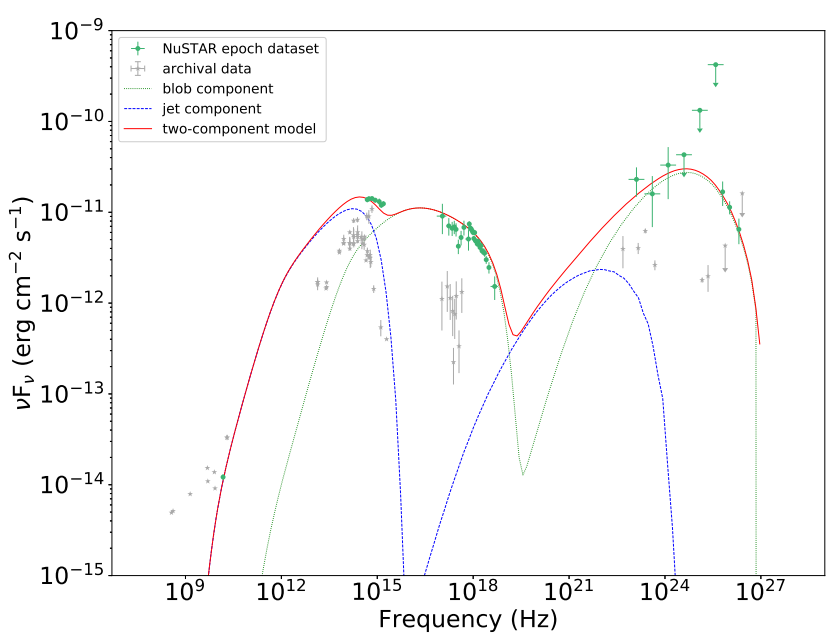

(b)

Figure 8. Broadband SED and modelled spectra for TXS 1515-273. The SED has been modelled considering the VLBA SED point in the radio band, KVA, Swift and NuSTAR SED points in the optical and X-ray energy band and the Fermi-LAT and MAGIC SED points in the gamma-ray band

, using a one-zone model (left) and a two-component model (right). Only Swift data simultaneous to NuSTAR observations were used. Coloured points represent observations in the different energy bands during the observations of 2019, grey points represent archival data. The red solid line represents the models, the green dotted line represents the blob emission and the blue dashed line represents the jet emission. Further details can be found in the text. For parameters, see Table 5.

In the two-component model instead the jet itself is in equipartition, but within the constraints on emission region size and magnetic field strength we derived from the X-ray observations (assuming "typical" Doppler factors), we did not find solutions where the blob would be in equipartition. We note that Tavecchio and Ghisellini (2016) found a two-component model solution for the low state SED of Mrk421 where the blob itself was in equipartition. However, this seems not to always be possible in the case of flares (see also MAGIC Collaboration et al. 2019).

\section{ACKNOWLEDGEMENTS}

We would like to thank the Instituto de Astrofísica de Canarias for the excellent working conditions at the Observatorio del Roque de los Muchachos in La Palma. The financial support of the German BMBF, MPG and HGF; the Italian INFN and INAF; the Swiss National Fund SNF; the ERDF under the Spanish Ministerio de Ciencia e Innovación (MICINN) (FPA2017-87859-P, FPA201785668-P, FPA2017-82729-C6-5-R, FPA2017-90566-REDC, PID2019-104114RB-C31, PID2019-104114RB-C32, PID2019105510GB-C31,PID2019-107847RB-C41, PID2019-107847RB- 
Table 5. SED modelling parameters for one-zone SSC and two-component models. Parameters are reported for the two available X-ray data set. See the text for the description of the parameters and the models.

\begin{tabular}{|c|c|c|c|c|c|c|c|c|c|c|c|c|}
\hline $\begin{array}{l}\text { X-ray } \\
\text { dataset }\end{array}$ & Model & Component & $\underset{\left(\times 10^{3}\right)}{\gamma_{\min }}$ & $\begin{array}{c}\gamma_{\mathrm{b}} \\
\left(\times 10^{4}\right)\end{array}$ & $\begin{array}{c}\gamma_{\max } \\
\left(\times 10^{5}\right)\end{array}$ & $n_{1}$ & $n_{2}$ & $\begin{array}{c}B \\
(\mathrm{G})\end{array}$ & $\begin{array}{c}K \\
\left(\times 10^{4} \mathrm{~cm}^{-3}\right)\end{array}$ & $\begin{array}{c}R \\
\left(\times 10^{15} \mathrm{~cm}\right)\end{array}$ & $\delta$ & $U_{\mathrm{B}} / U_{\mathrm{E}}$ \\
\hline \multirow{3}{*}{ XMM-Newton } & one-zone & - & 4.3 & 3.1 & 10.0 & 2.21 & 3.95 & 0.14 & 59 & 4.56 & 18 & $5 \times 10^{-3}$ \\
\hline & \multirow{2}{*}{ 2-component } & blob & 6.0 & 3.5 & 6.0 & 1.96 & 4.0 & 0.14 & 6.1 & 3.95 & 18 & $4 \times 10^{-3}$ \\
\hline & & jet & 0.52 & 0.52 & 0.19 & 1.74 & 2.84 & 0.14 & 0.0041 & 320 & 3.8 & 1.4 \\
\hline \multirow{3}{*}{ NuSTAR } & one-zone & - & 4.9 & 5 & 4.5 & 2.51 & 3.72 & 0.14 & 980 & 3.22 & 24 & $6 \times 10^{-3}$ \\
\hline & \multirow{2}{*}{ 2-component } & blob & 2.5 & 2.7 & 5.8 & 1.99 & 3.5 & 0.14 & 9.3 & 2.92 & 22 & $3 \times 10^{-3}$ \\
\hline & & jet & 0.52 & 0.52 & 0.19 & 1.74 & 2.84 & 0.14 & 0.0041 & 320 & 3.8 & 1.4 \\
\hline
\end{tabular}

C42, PID2019-107847RB-C44, PID2019-107988GB-C22); the Indian Department of Atomic Energy; the Japanese ICRR, the University of Tokyo, JSPS, and MEXT; the Bulgarian Ministry of Education and Science, National RI Roadmap Project DO1268/16.12.2019 and the Academy of Finland grant nr. 317637 and 320045 are gratefully acknowledged. This work was also supported by the Spanish Centro de Excelencia "Severo Ochoa" SEV-20160588, SEV-2017-0709 and CEX2019-000920-S, and "María de Maeztu" CEX2019-000918-M, the Unidad de Excelencia "María de Maeztu" MDM-2015-0509-18-2 and the "la Caixa" Foundation (fellowship LCF/BQ/PI18/11630012), by the Croatian Science Foundation (HrZZ) Project IP-2016-06-9782 and the University of Rijeka Project 13.12.1.3.02, by the DFG Collaborative Research Centers SFB823/C4 and SFB876/C3, the Polish National Research Centre grant UMO-2016/22/M/ST9/00382 and by the Brazilian MCTIC, CNPq and FAPERJ.

The Fermi-LAT Collaboration acknowledges generous ongoing support from a number of agencies and institutes that have supported both the development and the operation of the LAT as well as scientific data analysis. These include the National Aeronautics and Space Administration and the Department of Energy in the United States, the Commissariat à l'Energie Atomique and the Centre National de la Recherche Scientifique/Institut National de Physique Nucléaire et de Physique des Particules in France, the Agenzia Spaziale Italiana and the Istituto Nazionale di Fisica Nucleare in Italy, the Ministry of Education, Culture, Sports, Science and Technology (MEXT), High Energy Accelerator Research Organization (KEK) and Japan Aerospace Exploration Agency (JAXA) in Japan, and the K. A. Wallenberg Foundation, the Swedish Research Council and the Swedish National Space Board in Sweden. Additional support for science analysis during the operations phase from the following agencies is also gratefully acknowledged: the Istituto Nazionale di Astrofisica in Italy and and the Centre National d'Etudes Spatiales in France.

This research has made use of data and/or software provided by the High Energy Astrophysics Science Archive Research Center (HEASARC), which is a service of the Astrophysics Science Division at NASA/GSFC. This research has made use of data from the MOJAVE database that is maintained by the MOJAVE team (Lister et al. 2018).

\section{DATA AVAILABILITY}

The data underlying this article will be shared on reasonable request to the corresponding author.

\section{REFERENCES}

Aartsen, M. G. et al. (2020). Time-Integrated Neutrino Source Searches with 10 Years of IceCube Data. Phys. Rev. Lett., 124(5):051103.

Aartsen, M. G. et al. (2018a). Multimessenger observations of a flaring blazar coincident with high-energy neutrino IceCube-170922A. Science, 361(6398): eaat1378.

Aartsen, M. G. et al. (2018b). Neutrino emission from the direction of the blazar TXS 0506+056 prior to the IceCube-170922A alert. Science, 361(6398):147-151.

Abdo, A. A. et al. (2010). The Spectral Energy Distribution of Fermi Bright Blazars. ApJ, 716(1):30-70.

Abdollahi, S. et al. (2020). Fermi Large Area Telescope Fourth Source Catalog. ApJS, 247(1):33.

Acero, F. et al. (2016). Development of the Model of Galactic Interstellar Emission for Standard Point-source Analysis of Fermi Large Area Telescope Data. ApJS, 223(2):26.

Acero, F. et al. (2015). Fermi Large Area Telescope Third Source Catalog. Astrophys. J. Suppl., 218(2):23.

Ackermann, M. et al. (2011). The Second Catalog of Active Galactic Nuclei Detected by the Fermi Large Area Telescope. ApJ, 743(2):171.

Ackermann, M. et al. (2015). The Third Catalog of Active Galactic Nuclei Detected by the Fermi Large Area Telescope. ApJ, 810(1):14.

Ackermann, M. et al. (2016). 2FHL: The Second Catalog of Hard FermiLAT Sources. ApJS, 222(1):5.

Ackermann, M. et al. (2013). The First Fermi-LAT Catalog of Sources Above $10 \mathrm{GeV}$. Astrophys. J. Suppl., 209:34.

Aharonian, F. (2000). Tev gamma rays from bl lac objects due to synchrotron radiation of extremely high energy protons. New Astronomy, 5(7):377 -395 .

Aharonian, F. and Taylor, A. M. (2010). Limitations on the photodisintegration process as a source of VHE photons. Astroparticle Physics, 34(5):258-266.

Ahnen, M. et al. (2017). Performance of the magic telescopes under moonlight. Astroparticle Physics, 94:29 - 41.

Ajello, M. et al. (2017). 3FHL: The Third Catalog of Hard Fermi-LAT Sources. ApJS, 232(2): 18 .

Aleksić, J. et al. (2016). The major upgrade of the MAGIC telescopes, Part II: A performance study using observations of the Crab Nebula. Astroparticle Physics, 72:76-94.

Ansoldi, S. et al. (2018). The Blazar TXS 0506+056 Associated with a High-energy Neutrino: Insights into Extragalactic Jets and Cosmic-Ray Acceleration. ApJ, 863(1):L10.

Attridge, J. M. et al. (1999). Radio Jet-Ambient Medium Interactions on Parsec Scales in the Blazar 1055+018. ApJ, 518(2):L87-L90.

Atwood, W. B. et al. (2009). The Large Area Telescope on the Fermi Gamma-Ray Space Telescope Mission. ApJ, 697(2):1071-1102.

Barthelmy, S. D. et al. (2005). The Burst Alert Telescope (BAT) on the Swift MIDEX mission. Space Sci. Rev., 120:143.

Becerra González, J. et al. (2020). Optical spectral characterization of the the gamma-ray blazars S4 0954+65, TXS 1515-273 and RX J0812.0+0237. arXiv e-prints, page arXiv:2010.14532.

Bertero, M. (1989). Linear inverse and iii-posed problems. volume 75 of 
Advances in Electronics and Electron Physics, pages 1 - 120. Academic Press.

Bhatta, G. et al. (2018). Hard X-ray properties of NuSTAR blazars. $A \& A$, 619:A93.

Biteau, J. et al. (2020). Progress in unveiling extreme particle acceleration in persistent astrophysical jets. Nat. Astron., 4(2):124-131.

Bonnoli, G. et al. (2015). An emerging population of BL Lacs with extreme properties: towards a class of EBL and cosmic magnetic field probes? Monthly Notices of the Royal Astronomical Society, 451(1):611-621.

Breeveld, A. A. et al. (2010). Further calibration of the swift ultraviolet/optical telescope. Monthly Notices of the Royal Astronomical Society, 406(3):1687-1700.

Burrows, D. N. et al. (2005). The Swift X-ray Telescope. Space Sci. Rev., 120:165.

Cardelli, J. A. et al. (1989). The Relationship between Infrared, Optical, and Ultraviolet Extinction. ApJ, 345:245.

Cerruti, M. et al. (2019). Leptohadronic single-zone models for the electromagnetic and neutrino emission of TXS 0506+056. Mon. Not. Roy. Astron. Soc., 483(1):L12-L16.

Cerruti, M. et al. (2015). A hadronic origin for ultra-high-frequency-peaked BL Lac objects. MNRAS, 448(1):910-927.

Coppi, P. S. and Aharonian, F. A. (1999). Simultaneous X-ray and gammaray observations of $\mathrm{t}[\mathrm{CLC}] \mathrm{e}[/ \mathrm{CLC}] \mathrm{v}$ blazars: Testing synchro-compton emission models and probing the infrared extragalactic background. The Astrophysical Journal, 521(1):L33-L36.

Costamante, L. and Ghisellini, G. (2002). TeV candidate BL Lac objects. $A \& A, 384: 56-71$

Costamante, L. et al. (2001). Extreme synchrotron BL Lac objects. Stretching the blazar sequence. $A \& A, 371: 512-526$.

Cutini, S. (2019). Fermi-LAT detection of a GeV flare from highsynchrotron-peaked blazar TXS 1515-273. The Astronomer's Telegram, 12532:1.

Den Herder, J. W. et al. (2001). The reflection grating spectrometer on board xmm-newton. A\&A, 365(1):L7-L17.

Domínguez, A. et al. (2011). Extragalactic background light inferred from AEGIS galaxy-SED-type fractions. MNRAS, 410(4):2556-2578.

Evans, P. A. et al. (2009). Methods and results of an automatic analysis of a complete sample of Swift-XRT observations of GRBs. MNRAS, 397:1177-1201.

Fallah Ramazani, V. et al. (2017). Empirical multi-wavelength prediction method for very high energy gamma-ray emitting BL Lacertae objects. A\&A, 608:A68.

Foffano, L. et al. (2019). A new hard X-ray-selected sample of extreme highenergy peaked BL Lac objects and their TeV gamma-ray properties. Monthly Notices of the Royal Astronomical Society, 486(2):1741-1762.

Fossati, G. et al. G. (1998). A unifying view of the spectral energy distributions of blazars. MNRAS, 299(2):433-448.

Fukugita, M. et al. (1995). Galaxy Colors in Various Photometric Band Systems. PASP, 107:945.

Gehrels, N. et al. (2004). The Swift Gamma-Ray Burst Mission. ApJ, 611(2):1005-1020.

Georganopoulos, M. and Kazanas, D. (2003). Decelerating flows in TeV blazars: A resolution to the BL lacertae-FR i unification problem. The Astrophysical Journal, 594(1):L27-L30.

Ghisellini, G. (1999). Extreme blazars. Astroparticle Physics, 11(1):11 18. TeV Astrophysics of Extragalactic Sources.

Ghisellini, G. et al.(2017). The Fermi blazar sequence. MNRAS, 469(1):255266.

Ghisellini, G. et al. (2005). Structured jets in TeV BL Lac objects and radiogalaxies. Implications for the observed properties. A\&A, 432(2):401410.

Giroletti, M. et al. (2004). Parsec-Scale Properties of Markarian 501. ApJ, 600(1):127-140.

Goldoni, P. et al. (2020). Optical spectroscopy of Blazars for the Cherenkov Telescope Array. arXiv e-prints, page arXiv:2012.05176.

Harrison, F. A. et al. (2013). The Nuclear Spectroscopic Telescope Array (NuSTAR) High-energy X-Ray Mission. ApJ, 770:103.

Jansen, F. et al. (2001). Xmm-newton observatory-i. the spacecraft and operations. $A \& A, 365(1)$ :L1-L6

Kaur, A. et al. (2018). New high-z BL lacs using the photometric method with swift and SARA. The Astrophysical Journal, 859(2):80.

Keenan, M. et al. (2020). The Relativistic Jet Dichotomy and the End of the Blazar Sequence. arXiv e-prints, page arXiv:2007.12661.

Keivani, A. et al. (2018). A multimessenger picture of the flaring blazar TXS 0506+056: Implications for high-energy neutrino emission and cosmic-ray acceleration. The Astrophysical Journal, 864(1):84.

Kirk, J. G. et al. (1998). Particle acceleration and synchrotron emission in blazar jets. A\&A, 333:452-458.

Krawczynski, H. et al. (2001). Simultaneous X-ray and TeV gamma-ray observations of the TeV blazar Markarian 421 during February and May 2000. Astrophys. J., 559:187-195.

Lefaucheur, J. and Pita, S. (2017). Research and characterisation of blazar candidates among the Fermi/LAT 3FGL catalogue using multivariate classifications. A\&A, 602:A86.

Lindfors, E. J. et al. (2016). Optical and radio variability of the northern VHE gamma-ray emitting BL Lacertae objects. A\&A, 593:A98.

Lister, M. L. et al. (2011). $\gamma$-Ray and Parsec-scale Jet Properties of a Complete Sample of Blazars From the MOJAVE Program. ApJ, 742(1):27.

Lister, M. L. et al. (2018). MOJAVE. XV. VLBA $15 \mathrm{GHz}$ total intensity and polarization maps of 437 parsec-scale AGN jets from 1996 to 2017. The Astrophysical Journal Supplement Series, 234(1):12.

Lister, M. L. et al. (2019). MOJAVE. XVII. jet kinematics and parent population properties of relativistically beamed radio-loud blazars. The Astrophysical Journal, 874(1):43.

MAGIC Collaboration, Acciari, V. A. et al. (2019). A fast, very-high-energy $\gamma$-ray flare from BL Lacertae during a period of multi-wavelength activity in June 2015. A\&A, 623:A175.

MAGIC Collaboration, Acciari, V. A. et al. (2020a). Unravelling the complex behavior of Mrk 421 with simultaneous X-ray and VHE observations during an extreme flaring activity in April 2013. arXiv e-prints, page arXiv:2001.08678.

MAGIC Collaboration, Acciari, V. A. et al. (2020b). Broadband characterisation of the very intense TeV flares of the blazar 1ES 1959+650 in 2016. $A \& A, 638: \mathrm{A} 14$

MAGIC Collaboration, Acciari, V. A. et al. (2020c). Testing two-component models on very-high-energy gamma-ray emitting BL Lac objects. arXiv e-prints, page arXiv:2006.04493.

Mannheim, K. (1993). The proton blazar. A\&A, 269:67-76.

Maraschi, L. et al. (1999). Simultaneous X-ray and t[CLC]e[/CLC]v observations of a rapid flare from Markarian 421. The Astrophysical Journal, 526(2):L81-L84.

Maraschi, L. and Tavecchio, F. (2003). The jet-disk connection and blazar unification. The Astrophysical Journal, 593(2):667-675.

Mirzoyan, R. (2019). Detection of sub-TeV gamma-ray emission from the flaring blazar TXS 1515-273 with the MAGIC telescopes. The Astronomer's Telegram, 12538:1.

Mücke, A. and Protheroe, R. J. (2001). A proton synchrotron blazar model for flaring in Markarian 501. Astroparticle Physics, 15(1):121-136.

Murase, K. et al. (2012). BLAZARS AS ULTRA-HIGH-ENERGY COSMIC-RAY SOURCES: IMPLICATIONS FOR TeV GAMMARAY OBSERVATIONS. The Astrophysical Journal, 749(1):63.

Nilsson, K. et al. (2018). Long-term optical monitoring of TeV emitting blazars. I. Data analysis. A\&A, 620:A185.

Nolan, P. L. et al. (2012). Fermi Large Area Telescope Second Source Catalog. ApJS, 199(2):31.

Oh, K. et al. (2018). The 105 month Swift-BAT all-sky hard X-ray survey. Astrophys. J. Suppl., 235(1):4.

Padovani, P. et al. (2017). Active galactic nuclei: what's in a name? Astron. Astrophys. Rev., 25(1):2.

Pian, E. et al. (1998). BeppoSAX Observations of Unprecedented Synchrotron Activity in the BL Lacertae Object Markarian 501. ApJ, 492(1):L17-L20.

Piner, B. G. and Edwards, P. G. (2018). Multi-epoch VLBA Imaging of 20 New TeV Blazars: Apparent Jet Speeds. ApJ, 853(1):68.

Poole, T. S. et al. (2008). Photometric calibration of the Swift ultraviolet/optical telescope. MNRAS, 383(2):627-645. 
Roming, P. W. A. et al. (2005). The Swift Ultra-Violet/Optical Telescope. Space Sci. Rev., 120:95-142.

Schlafly, E. F. and Finkbeiner, D. P. (2011). Measuring Reddening with Sloan Digital Sky Survey Stellar Spectra and Recalibrating SFD. ApJ, 737(2):103.

Stickel, M. et al. (1991). The Complete Sample of 1 Jansky BL Lacertae Objects. I. Summary Properties. ApJ, 374:431

Stocke, J. T. et al. (1991). The Einstein Observatory Extended MediumSensitivity Survey. II. The Optical Identifications. ApJS, 76:813.

Strüder, L. et al. (2001). The european photon imaging camera on xmmnewton: the pn-ccd camera. $A \& A, 365(1)$ :L18-L26.

Takahashi, T. et al. (2000). Complex spectral variability from intensive multiwavelength monitoring of Markarian 421 in 1998. The Astrophysical Journal, 542(2):L105-L109.

Tavecchio, F. et al. (2011). On the origin of the $\gamma$-ray emission from the flaring blazar PKS 1222+216. A\&A, 534:A86.

Tavecchio, F. and Ghisellini, G. (2015). On the magnetization of BL Lac jets. Monthly Notices of the Royal Astronomical Society, 456(3):2374-2382.

Tavecchio, F. and Ghisellini, G. (2016). On the magnetization of BL Lac jets. MNRAS, 456(3):2374-2382.

Tavecchio, F. et al. (2010). TeV BL Lac objects at the dawn of the Fermi era. Monthly Notices of the Royal Astronomical Society, 401(3):1570-1586.

Tavecchio, F. and Sobacchi, E. (2020). Anisotropic electron populations in BL Lac jets: consequences for the observed emission. MNRAS, 491(2):2198-2204.

Turner, M. J. L. et al. (2001). The european photon imaging camera on xmm-newton: the mos cameras. $A \& A, 365(1)$ :L27-L35.

Valtaoja, L. et al. (1991). UBVRI Photopolarimetry of BL Lac Objects: The Connection Between Frequency- Dependent Polarization and Growing Radio Shocks. AJ, 101:78

Wachter, K. et al. (1979). Parameter estimation in X-ray astronomy using maximum likelihood. ApJ, 230:274-287.

Willingale, R. et al. (2013). Calibration of X-ray absorption in our Galaxy. MNRAS, 431(1):394-404.

Zanin, R. et al. (2013). MARS, The MAGIC Analysis and Reconstruction Software. In International Cosmic Ray Conference, volume 33 of International Cosmic Ray Conference, page 2937.

Zhang, Y. et al. (2002). Four years monitoring of blazar pks 2155-304 with bepposax: probing the dynamics of the jet. Astrophys. J., 572:762.

\section{AFFILIATIONS}

(1) Instituto de Astrofisica de Canarias, La Laguna (Tenerife), Spain, (2) Università di Udine and INFN Trieste, Italy, Udine, Italy, (3) INAF - National Institute for Astrophysics, Roma, Italy, (4) ETH Zürich, Institute for Particle Physics, Zürich, Switzerland, (5) Institut de Física d'Altes Energies (IFAE), The Barcelona Institute of Science and Technology (BIST), E-08193 Bellaterra (Barcelona), Spain, Bellaterra (Barcelona), Spain, (6) Japanese MAGIC Group: ICRR, The University of Tokyo, Chiba, Japan, (7) Technische Universität Dortmund, Dortmund, Germany, (8) Croatian MAGIC Group: University of Zagreb, Faculty of Electrical Engineering and Computing (FER), Zagreb, Croatia, (9) IPARCOS Institute and EMFTEL Department, Universidad Complutense de Madrid, Madrid, Spain, (10) Centro Brasileiro de Pesquisas Físicas (CBPF), Rio de Janeiro, Brazil, (11) Dipartimento di Fisica e Astronomia dell? Università and Sezione INFN, Padova, Italy, Padova, Italy, (12) University of Lodz, Faculty of Physics and Applied Informatics, Department of Astrophysics, 90-236 Lodz, Poland, Lodz, Poland, (13) Dipartimento SFTA, Sezione di Fisica, Università di Siena and INFN sez. di Pisa, Siena, Italy, (14) Deutsches Elektronen-Synchrotron (DESY) Zeuthen, Zeuthen, Germany, (15) INFN MAGIC Group: INFN Sezione di Torino and Università degli Studi di Torino, Torino, Italy, (16)
Max-Planck-Institut für Physik, München, München, Germany, (17) Universita di Pisa and INFN Pisa, Pisa, Italy, (18) Universitat de Barcelona, Barcelona, Spain, (19) Armenian MAGIC Group: A. Alikhanyan National Science Laboratory, Yerevan, Armenia, (20) Centro de Investigaciones Energeticas, Medioambientales y Tecnologicas, Madrid, Spain, (21) INFN MAGIC Group: INFN Sezione di Bari and Dipartimento Interateneo di Fisica dell'Università e del Politecnico di Bari, Bari, Italy, (22) Croatian MAGIC Group: University of Rijeka, Department of Physics, Rijeka, Croatia, (23) Institut für Theoretische Physik und Astrophysik, Fakultät für Physik und Astronomie, Universität Würzburg, Würzburg, Germany, (24) Finnish MAGIC Group: Finnish Centre for Astronomy with ESO, University of Turku, Turku, Finland, (25) Universitat Autònoma de Barcelona, Barcelona, Spain, (26) Armenian MAGIC Group: ICRANet-Armenia at NAS RA, Yerevan, Armenia, (27) Croatian MAGIC Group: University of Split, Faculty of Electrical Engineering, Mechanical Engineering and Naval Architecture (FESB), Split, Croatia, (28) Croatian MAGIC Group: Josip Juraj Strossmayer University of Osijek, Department of Physics, Osijek, Croatia, (29) Japanese MAGIC Group: RIKEN, Saitama, Japan, (30) Japanese MAGIC Group: Department of Physics, Kyoto University, Kyoto, Japan, (31) Japanese MAGIC Group: Department of Physics, Tokai University, Kanagawa, Japan, (32) Saha Institute of Nuclear Physics, HBNI, Kolkata, India, (33) Institute for Nuclear Research and Nuclear Energy, Sofia, Bulgaria, (34) Finnish MAGIC Group: Astronomy Research Unit, University of Oulu, Oulu, Finland, (35) Croatian MAGIC Group: Ru?er Bo?kovi? Institute, Zagreb, Croatia, (36) INFN MAGIC Group: INFN Sezione di Perugia, Perugia, Italy, (37) INFN MAGIC Group: INFN Roma Tor Vergata, Roma, Italy, (38) now at University of Innsbruck, (39) also at Port d'Informació Científica (PIC) E-08193 Bellaterra (Barcelona) Spain, (40) now at Ruhr-Universität Bochum, Fakultät für Physik und Astronomie, Astronomisches Institut (AIRUB), 44801 Bochum, Germany, (41) also at Dipartimento di Fisica, Università di Trieste, I-34127 Trieste, Italy, (42) Max-Planck-Institut für Physik, D-80805 München, Germany, (43) also at INAF Trieste and Dept. of Physics and Astronomy, University of Bologna, (44) Japanese MAGIC Group: Institute for Cosmic Ray Research (ICRR), The University of Tokyo, Kashiwa, 277-8582 Chiba, Japan), (45) Dipartimento Interateneo di Fisica dell'Università e del Politecnico di Bari, 70125 Bari, Italy, (46), Dipartimento Interateneo di Fisica dell'Università e del Politecnico di Bari, 70125 Bari, Italy, (47) Instituto de Astrofísica de Andalucía (CSIC), Apartado 3004, E-18080 Granada, Spain, (48) INFN Sezione di Perugia, 06123 Perugia, Italy, (49) INAF - Istituto di Radioastronomia, Via Gobetti 101, I-40129 Bologna, Italy, (50) Department of Physics and Astronomy, University of Turku, FI-20014, Turku, Finland, (51) Università di Siena, I-53100, Siena, Italy

\section{APPENDIX A: DETAILS OF THE MULTI-WAVELENGTH ANALYSIS.}

Here we provide more detail on the observations and the analysis performed in the different energy ranges.

This paper has been typeset from a $\mathrm{T}_{\mathrm{E}} \mathrm{X} / \mathrm{LT}_{\mathrm{E}} \mathrm{X}$ file prepared by the author. 
Table A1. Observed flux of TXS 1515-273 observation with MAGIC. The $95 \%$ confidence level upper limits are also reported for observations with less than $3 \sigma$ significance.

\begin{tabular}{ccccc}
\hline MJD & Time Flux $(>400 \mathrm{GeV})$ & Flux $(>400 \mathrm{GeV})$ & UL Significance \\
& $(\mathrm{h})$ & $\left(\times 10^{-12} \mathrm{~cm}^{-2} \mathrm{~s}^{-1}\right)$ & $\left(\times 10^{-12} \mathrm{~cm}^{-2} \mathrm{~s}^{-1}\right)$ & $\sigma$ \\
\hline 58541.21 & 1.31 & $6.28 \pm 2.16$ & & 3 \\
58542.27 & 1.97 & $7.42 \pm 1.29$ & & 7 \\
58543.20 & 1.31 & - & 4.47 & 0 \\
58544.19 & 0.35 & - & 1.79 & 0.7 \\
58545.22 & 2.12 & $3.85 \pm 1.19$ & & 5 \\
58547.19 & 1.04 & - & 4.65 & 1 \\
\hline
\end{tabular}

Table A2. Observed flux of TXS 1515-273 observation with Fermi-LAT. The exposure time for each bin is $24 \mathrm{~h}$, and the MJD values reported in the first column correspond to the middle of the time bin. The $95 \%$ confidence level upper limits are reported for bins with $\mathrm{TS}<9$.

\begin{tabular}{cccc}
\hline MJD & \multicolumn{3}{c}{ Flux $(>300 \mathrm{MeV})$} \\
& $\left(\times 10^{-7} \mathrm{~cm}^{-2} \mathrm{~s}^{-1}\right)$ & $\left(\times 10^{-7} \mathrm{~cm}^{-2} \mathrm{~s}^{-1}\right)$ & \\
\hline 58539.22 & $0.635 \pm 0.328$ & & $\mathrm{TS}$ \\
58540.22 & $0.821 \pm 0.371$ & & 9.36 \\
58541.22 & $0.674 \pm 0.316$ & & 11.75 \\
58542.22 & $0.549 \pm 0.296$ & & 22.00 \\
58543.22 & - & 0.228 & 11.35 \\
58544.22 & $0.511 \pm 0.294$ & & 0 \\
58545.22 & - & 1.266 & 10.62 \\
58546.22 & $4.664 \pm 0.842$ & & 6.74 \\
58547.22 & - & 0.192 & 138.29 \\
58548.22 & - & 1.020 & 0.97 \\
\hline
\end{tabular}

Table A3. $\log$ and fitting results of XMM-Newton observations of TXS 1515-273 with $N_{\mathrm{H}}$ fixed to Galactic absorption. Fluxes are corrected for the Galactic absorption.

\begin{tabular}{cccc}
\hline MJD & $\begin{array}{c}\text { Exposure time } \\
(\mathrm{ks})\end{array}$ & $\begin{array}{c}\text { Flux }(3-10 \mathrm{keV}) \\
\left(\times 10^{-12} \mathrm{erg} \mathrm{cm}^{-2} \mathrm{~s}^{-1}\right)\end{array}$ & $\begin{array}{c}\text { Flux }(0.3-10 \mathrm{keV}) \\
\left(\times 10^{-12} \mathrm{erg} \mathrm{cm}^{-2} \mathrm{~s}^{-1}\right)\end{array}$ \\
\hline 58544.10 & 25 & $2.47 \pm 0.02$ & $8.16 \pm 0.05$ \\
\hline
\end{tabular}

Table A4. Log and fitting results of NuSTAR observations of TXS 1515-273 with $N_{\mathrm{H}}$ fixed to Galactic absorption. Fluxes are corrected for the Galactic absorption.

\begin{tabular}{cccc}
\hline MJD & $\begin{array}{c}\text { Exposure time } \\
(\mathrm{ks})\end{array}$ & $\begin{array}{c}\text { Flux }(3-10 \mathrm{keV}) \\
\left(\times 10^{-12} \mathrm{erg} \mathrm{cm}^{-2} \mathrm{~s}^{-1}\right)\end{array}$ & $\begin{array}{c}\text { Flux }(4-79 \mathrm{keV}) \\
\left(\times 10^{-12} \mathrm{erg} \mathrm{cm}^{-2} \mathrm{~s}^{-1}\right)\end{array}$ \\
\hline 58544.95 & 34 & $5.69 \pm 0.04$ & $3.25 \pm 0.02$ \\
\hline
\end{tabular}

Table A5. Log and fitting results of Swift-XRT observations of TXS 1515273 using a PL model with $N_{\mathrm{H}}$ fixed to Galactic absorption. Fluxes are corrected for the Galactic absorption.

\begin{tabular}{cccc}
\hline MJD & $\begin{array}{c}\text { Exposure time } \\
(\mathrm{s})\end{array}$ & $\begin{array}{c}\text { Flux 0.3-10 keV } \\
\left(\times 10^{-12} \mathrm{erg} \mathrm{cm}^{-2} \mathrm{~s}^{-1}\right)\end{array}$ & $\begin{array}{c}\text { Photon index } \\
\left(\Gamma_{\mathrm{X}}\right)\end{array}$ \\
\hline 56930.87 & 1193.7 & $1.08 \pm 0.20$ & $2.84 \pm 0.32$ \\
58488.34 & 229.8 & $5.37 \pm 0.11$ & $2.55 \pm 0.30$ \\
58541.65 & 2462.3 & $1.69 \pm 0.65$ & $2.14 \pm 0.51$ \\
58544.12 & 536.6 & $8.16 \pm 0.81$ & $2.50 \pm 0.14$ \\
58544.84 & 401.2 & $1.78 \pm 0.21$ & $2.00 \pm 0.15$ \\
58544.97 & 529.0 & $1.75 \pm 0.19$ & $2.29 \pm 0.15$ \\
58547.43 & 2497.3 & $3.83 \pm 0.23$ & $2.73 \pm 0.10$ \\
58551.62 & 1648.2 & $2.73 \pm 0.26$ & $2.89 \pm 0.14$ \\
58554.60 & 2582.2 & $5.24 \pm 0.29$ & $2.61 \pm 0.92$ \\
58560.71 & 2467.3 & $2.38 \pm 0.17$ & $2.88 \pm 0.13$ \\
\hline
\end{tabular}

Table A6. Observed magnitudes with XMM-OM.

\begin{tabular}{cccc}
\hline MJD & $\begin{array}{c}\text { B } \\
(\mathrm{AB} \mathrm{mag})\end{array}$ & $\begin{array}{c}\mathrm{U} \\
(\mathrm{AB} \text { mag })\end{array}$ & $\begin{array}{c}\text { W1 } \\
(\mathrm{AB} \mathrm{mag})\end{array}$ \\
\hline 58544.10 & $16.639 \pm 0.005$ & $17.089 \pm 0.008$ & $17.406 \pm 0.009$ \\
\hline
\end{tabular}

Table A7. Observed magnitudes for TXS 1515-273 with Siena Observatory.

\begin{tabular}{cc}
\hline MJD & $\begin{array}{c}\text { Observed magnitude } \\
(\mathrm{mag})\end{array}$ \\
\hline 58542.17 & $15.43 \pm 0.02$ \\
58551.14 & $15.50 \pm 0.02$ \\
58564.10 & $15.56 \pm 0.03$ \\
\hline
\end{tabular}


Table A8. Observed magnitudes with KVA.

\begin{tabular}{cc}
\hline MJD & Observed magnitude \\
& $(\mathrm{mag})$ \\
\hline 58541.22 & $15.46 \pm 0.02$ \\
58542.21 & $15.42 \pm 0.02$ \\
58543.23 & $15.5 \pm 0.02$ \\
58544.24 & $15.43 \pm 0.06$ \\
58545.24 & $15.44 \pm 0.02$ \\
58547.23 & $15.39 \pm 0.02$ \\
58549.24 & $15.45 \pm 0.02$ \\
58551.22 & $15.48 \pm 0.02$ \\
58552.24 & $15.54 \pm 0.02$ \\
58554.21 & $15.48 \pm 0.02$ \\
58555.2 & $15.39 \pm 0.02$ \\
58556.2 & $15.46 \pm 0.02$ \\
58557.19 & $15.51 \pm 0.02$ \\
58558.17 & $15.52 \pm 0.02$ \\
58559.24 & $15.57 \pm 0.02$ \\
58560.22 & $15.66 \pm 0.08$ \\
58565.25 & $15.68 \pm 0.03$ \\
58580.18 & $15.78 \pm 0.02$ \\
58584.2 & $15.8 \pm 0.03$ \\
58586.19 & $15.76 \pm 0.02$ \\
58596.14 & $15.99 \pm 0.04$ \\
58600.16 & $15.92 \pm 0.03$ \\
58603.14 & $15.93 \pm 0.03$ \\
58609.95 & $16.0 \pm 0.06$ \\
58617.12 & $16.05 \pm 0.04$ \\
58633.9 & $15.89 \pm 0.03$ \\
58643.93 & $16.11 \pm 0.03$ \\
58658.97 & $16.14 \pm 0.03$ \\
58666.94 & $16.14 \pm 0.03$ \\
58673.92 & $16.0 \pm 0.03$ \\
58678.91 & $16.08 \pm 0.04$ \\
58687.9 & $15.95 \pm 0.03$ \\
58692.91 & $15.98 \pm 0.03$ \\
58699.92 & $16.03 \pm 0.03$ \\
58706.89 & $16.0 \pm 0.04$ \\
58714.88 & $15.93 \pm 0.03$ \\
58718.87 & $15.86 \pm 0.03$ \\
\hline &
\end{tabular}


Table A9. Observed magnitudes for TXS 1515-273 obtained with Swift-UVOT.

\begin{tabular}{ccccccc}
\hline MJD & $\begin{array}{c}\mathrm{V} \\
(\mathrm{mag})\end{array}$ & $\begin{array}{c}\mathrm{B} \\
(\mathrm{mag})\end{array}$ & $\begin{array}{c}\mathrm{U} \\
(\mathrm{mag})\end{array}$ & $\begin{array}{c}\mathrm{W} 1 \\
(\mathrm{mag})\end{array}$ & $\begin{array}{c}\text { M2 } \\
(\mathrm{mag})\end{array}$ & $\begin{array}{c}\text { W2 } \\
(\mathrm{mag})\end{array}$ \\
\hline 56930 & $16.74 \pm 0.14$ & $17.72 \pm 0.16$ & $16.96 \pm 0.12$ & $17.50 \pm 0.16$ & $17.71 \pm 0.14$ & $17.74 \pm 0.12$ \\
58488 & - & - & $16.58 \pm 0.07$ & - & - & - \\
58541 & $15.95 \pm 0.06$ & $16.56 \pm 0.06$ & $15.97 \pm 0.06$ & $16.19 \pm 0.07$ & $16.60 \pm 0.09$ & $16.52 \pm 0.07$ \\
58544 & $15.89 \pm 0.07$ & $16.60 \pm 0.06$ & $15.92 \pm 0.07$ & $16.23 \pm 0.08$ & $16.67 \pm 0.10$ & $16.58 \pm 0.07$ \\
58547 & $15.87 \pm 0.05$ & $16.49 \pm 0.05$ & $15.88 \pm 0.06$ & $16.25 \pm 0.07$ & $16.58 \pm 0.08$ & $16.60 \pm 0.07$ \\
58551 & $16.07 \pm 0.07$ & $16.59 \pm 0.06$ & $16.01 \pm 0.07$ & $16.36 \pm 0.08$ & $16.70 \pm 0.09$ & $16.63 \pm 0.08$ \\
58554 & $15.87 \pm 0.05$ & $16.52 \pm 0.05$ & $15.82 \pm 0.06$ & $16.24 \pm 0.07$ & $16.50 \pm 0.08$ & $16.52 \pm 0.07$ \\
58560 & $16.09 \pm 0.06$ & $16.77 \pm 0.06$ & $16.10 \pm 0.06$ & $16.37 \pm 0.08$ & $16.70 \pm 0.08$ & $16.84 \pm 0.07$ \\
\hline
\end{tabular}

